\title{
On a class of stochastic fractional kinetic equation with fractional noise
}

Min $\mathrm{Lu}^{1}$ and Junfeng $\mathrm{Liu}^{1 *}$

\section{"Correspondence:}

jordanjunfeng@163.com

'School of Statistics and

Mathematics, Nanjing Audit

University, Nanjing, P.R. China

\section{Springer}

\begin{abstract}
In this article we study a class of stochastic fractional kinetic equations with fractional noise which are spatially homogeneous and are fractional in time with $H>1 / 2$. The diffusion operator involved in the equation is the composition of the Bessel and Riesz potentials with any fractional parameters. We prove the existence of the solution under some mild conditions which generalized some results obtained by Dalang (Electron. J. Probab. 4(6):1-29, 1999) and Balan and Tudor (Stoch. Process. Appl. 120:2468-2494, 2010). We study also its Hölder continuity with respect to space and time variables with $b=0$. Moreover, we prove the existence for the density of the solution and establish the Gaussian-type lower and upper bounds for the density by the techniques of Malliavin calculus.
\end{abstract}

MSC: $60 \mathrm{G} 35 ; 60 \mathrm{H} 15 ; 60 \mathrm{H} 07$

Keywords: Stochastic fractional kinetic equation; Bessel and Riesz potentials; Fractional noise; Malliavin calculus; Density estimates

\section{Introduction}

This paper is concerned with the following stochastic fractional kinetic equation (SFKE for short) with zero initial condition (see, for example, Angulo et al. [1, 3], Angulo et al. [2], and Márquez-Carreras [16]):

$$
\left\{\begin{array}{l}
\frac{\partial}{\partial t} u(t, x)=-(I-\Delta)^{\frac{\alpha}{2}}(-\Delta)^{\frac{\gamma}{2}} u(t, x)+b(u(t, x))+\dot{W}(t, x) \quad \text { in }[0, T] \times \mathbb{R}^{d}, \\
u(0, x)=0, \quad x \in \mathbb{R}^{d}
\end{array}\right.
$$

with $T>0, d \geq 1, \alpha \geq 0, \gamma>0, b(\cdot)$ is a measurable function and $\dot{W}$ denotes a fractional noise. We will specify later the required conditions on the noise $\dot{W}$. In the SFKE (1), $I$ and $\Delta$ are the identity and Laplacian operators, respectively, and the operators $(I-\Delta)^{\frac{\alpha}{2}}$ and $(-\Delta)^{\frac{\gamma}{2}}$ have to be interpreted as the inverses of the Bessel potential and Riesz potential, respectively. The fractional Riesz-Bessel operator was introduced by Angulo et al. in [3] and the authors established the existence of the Riesz-Bessel motion.

The SFKE (1) (known also as Riesz-Bessel fractional diffusion equation, the fractional diffusion equation or the fractional heat equation) driven by Gaussian white noise was introduced by Angulo et al. [2] via the Eulerian approach. It was mainly used to model some

(c) The Author(s) 2021. This article is licensed under a Creative Commons Attribution 4.0 International License, which permits use, sharing, adaptation, distribution and reproduction in any medium or format, as long as you give appropriate credit to the original author(s) and the source, provide a link to the Creative Commons licence, and indicate if changes were made. The images or other third party material in this article are included in the article's Creative Commons licence, unless indicated otherwise in a credit line to the material. If material is not included in the article's Creative Commons licence and your intended use is not permitted by statutory regulation or exceeds the permitted use, you will need to obtain permission directly from the copyright holder. To view a copy of this licence, visit http://creativecommons.org/licenses/by/4.0/. 
physical phenomena, such as diffusion in porous media with fractal geometry, kinematics in viscoelastic media, and propagation of seismic waves. In [2], the authors mainly studied the SFKE (1) with additive Gaussian space-time white noise in bounded and unbounded spatial domains. They connected it with the Eulerian theory of turbulence dispersion by means of the advection-diffusion equation. They also gave a very interesting connection with the Lagrangian theory.

Nowadays we can find a lot of applications of these equations in turbulence, ecology, hydrology, geophysics, image processing, neurophysiology, economics and finance, etc. (see Angulo et al. [2], Anh et al. [4, 5], Márquez-Carreras [16] and the references therein for more details). The composition of the Bessel and Riesz potentials plays an important role in describing the behavior of the process at the spatial macro and microscales. Apart from the classical context of heat conduction, an equation of form the SFKE (1) with $\alpha=0$ and $\gamma=2$ also arises in neurophysiology; see [21] for example. Diffusion operators in the SFKE (1) with $\alpha=0$ and $\gamma>0$ correspond to the generalized heat equation which have been used to define hyperviscosity and to study its effect on the inertial-range scaling of fully developed turbulence [13]. The presence of the Bessel operator is essential for a study of stationary solutions of the SFKE (1). One can also see [4] and [5] for related models.

After the nice work of Angulo et al. [2], several authors have also studied this kind of the SFKE (1) and other similar equations from a mathematical point of view. For example, Angulo et al. [1] considered a more generalized type of space-time fractional kinetic equation with Gaussian white noise or infinitely divisible noise as follows:

$$
\left(A_{n} \frac{\partial^{\beta_{n}}}{\partial t^{\beta_{n}}}+\cdots+A_{1} \frac{\partial^{\beta_{1}}}{\partial t^{\beta_{1}}}+A_{0} \frac{\partial^{\beta_{0}}}{\partial t^{\beta_{0}}}\right) u(t, x)=-(I-\Delta)^{\frac{\alpha}{2}}(-\Delta)^{\frac{\gamma}{2}} u(t, x)+\dot{X}(t, x),
$$

with $\beta_{n}>\beta_{n-1}>\cdots>\beta_{1}>\beta_{0} \geq 0, A_{i}>0, i=0, \ldots, n$, and the fractional-in-time derivative is defined in the Caputo-Djrbashian sense, i.e.,

$$
\frac{\partial^{\beta} u}{\partial t^{\beta}}(t, x)= \begin{cases}\frac{\partial^{m} u}{\partial t^{m}}(t, x) & \text { if } \beta=m \in \mathbb{N} \\ \frac{1}{\Gamma(m-\beta)} \int_{0}^{t}(t-r)^{m-\beta-1} \frac{\partial^{m} u(r, x)}{\partial r^{m}} d r & \text { if } m-1<\beta<m\end{cases}
$$

where $\Gamma(\cdot)$ is the gamma function. The solutions to the equation are proved in both bounded and unbounded domains, in conjunction with bounds for the variances of the increments. The role of each of the parameters in the equation is investigated with respect to second- and higher-order properties. In particular, they also proved that the long-range dependence may arise in the temporal solution under certain conditions on the spatial operators. In [6], the authors provided a detailed review of the related literature. They considered a more general class of fractional (both in time and space) evolution equation defined on Dirichlet regular bounded open domains. They derived the sufficient conditions for the definition of a weak-sense Gaussian solution. The Hölder regularity of the solution with respect to the time and space variables is also derived.

Meanwhile Márquez-Carreras [15] dealt with the SFKE (1) driven by a Gaussian noise which is white in time and correlated in space. They proved the existence and uniqueness of solution by means of a weak formulation and studied the Hölder continuity of this solution. Moreover, they also proved the existence of a smooth density associated to the 
solution process and studied the asymptotic behavior of this density. Later on MárquezCarreras [16] studied the following kind of stochastic partial differential equations:

$$
\frac{\partial}{\partial t} u(t, x)=-(I-\Delta)^{\frac{\alpha}{2}} u(t, x)+a(u(t, x))+b(u(t, x)) \dot{W}(t, x) \quad \text { with }[0, T] \times \mathbb{R}^{d},
$$

with $\alpha>0$ and the process $\dot{W}$ is a Gaussian noise, white in time and correlated in space. The existence and uniqueness of solution and the Hölder continuity of this solution was proved. Moreover, they proved the existence of the density of the solution and that its density was smooth.

In this paper, regarding the structure of the SFKE (1), we prove the existence and uniqueness of the solution and the Hölder continuity of this solution. Moreover, we show that the equation of the solution is absolutely continuous with respect to Lebesgue's measure on $\mathbb{R}^{d}$ (with $d<\alpha+\gamma$ ) and establish the lower and upper bounds for its density by means of Malliavin calculus.

We would like to list some differences between this study and all the papers mentioned above. Firstly, the SFKE (1) we considered in this paper is driven by a more general Gaussian noise (fractional in time and correlated in space) which extended the former noises in Angulo et al. [2], Angulo et al. [1], and Márquez-Carreras [16]. Secondly, thanks to the fractional noise, the properties of the solution are checked for any $\alpha>0$ and $\gamma>0$ and not for a more restricted region. Moreover, these properties do not depend on the dimension of $x$. Finally, we generalize some results of Balan and Tudor $[7,8]$ to the fractional operator setting. We study some new properties of the mild solution to the SFKE (1). Here, we deal widely with the Hölder continuity in time and in space. We also study some density properties of the solution by using the techniques of Malliavin calculus; see, for example Nualart and Quer-Sardanyons [19, 20], and Liu and Yan [14].

This article is organized as follows. In Sect. 2 we recall some preliminaries including the fractional noise and Malliavin calculus. Section 3 is devoted to describe what we understand by a solution of the SFKE (1) and prove the existence and uniqueness of this solution. We show that the solution of the SFKE (1) exists if (12) holds. In Sect. 4 we check that spatially the solution of the SFKE (1) with $b=0$ is a Gaussian field with zero mean, stationary increments, and a continuous covariance function. We find its index (see Definition 4.1). We also show that the solution is not stationary in time. Finally in Sect. 5 we study the density properties of the solution of the SFKE (1), such as the existence of the density and related Gaussian-type lower and upper bounds for the density.

\section{The preliminaries}

This section is devoted to recalling some preliminaries about the fractional noise and related Malliavin calculus.

\subsection{Fractional noise}

Let us start by introducing some basic notions on Fourier transforms of functions: the space of real valued infinitely differentiable functions with compact support is denoted by $\mathcal{D}\left(\mathbb{R}^{d}\right)$ and by $\mathcal{S}\left(\mathbb{R}^{d}\right)$ the Schwartz space of rapidly decreasing $C^{\infty}$ functions in $\mathbb{R}^{d}$. For 
$\varphi \in L^{1}\left(\mathbb{R}^{d}\right)$, we let $\mathcal{F} \varphi$ be the Fourier transform of $\varphi$

$$
\mathcal{F} \varphi(\xi)=\int_{\mathbb{R}^{d}} e^{-\mathrm{i} \xi \cdot x} \varphi(x) d x
$$

so that the inverse Fourier transform is given by $\mathcal{F}^{-1} \varphi(\xi)=(2 \pi)^{-d} \mathcal{F} \varphi(-\xi)$.

Similarly to [8] or [11] for the general case, on a complete probability space $\left(\Omega, \mathcal{F}, \mathscr{F}_{t}, P\right)$, for $H>1 / 2$, we consider a zero-mean Gaussian process $W=\left\{W(\varphi), \varphi \in \mathcal{D}\left([0, T] \times \mathbb{R}^{d}\right)\right\}$ with covariance given by

$$
\mathbb{E}(W(\varphi) W(\phi))=\alpha_{H} \int_{0}^{T} \int_{0}^{T} \int_{\mathbb{R}^{2 d}} \varphi(t, x) \phi(s, y)|t-s|^{2 H-2} \Lambda(x-y) d x d y d s d t
$$

with $\alpha_{H}=H(2 H-1)$ and $\Lambda(\cdot): \mathbb{R}^{d} \rightarrow \mathbb{R}_{+}$is a non-negative definite function and its Fourier transform $\mathcal{F} \Lambda=\mu$ is a tempered measure. Moreover, we assume that there is an integer $m \geq 1$ such that

$$
\int_{\mathbb{R}^{d}} \frac{1}{\left(1+|\xi|^{2}\right)^{m}} \mu(d \xi)<\infty
$$

We call $\dot{W}$ the fractional noise; it has a spatial covariance $\Lambda(\cdot)$ and has the covariance of a fractional Brownian motion with Hurst parameter $H>1 / 2$ in time.

Let $\mathcal{H}$ be the completion of $\mathcal{D}\left([0, T] \times \mathbb{R}^{d}\right)$ endowed with the inner product

$$
\begin{aligned}
\langle\varphi, \phi\rangle_{\mathcal{H}} & =\alpha_{H} \int_{0}^{T} \int_{0}^{T} \int_{\mathbb{R}^{2 d}} \varphi(t, x) \phi(s, y)|t-s|^{2 H-2} \Lambda(x-y) d x d y d s d t \\
& =\alpha_{H} \int_{0}^{T} \int_{0}^{T} \int_{\mathbb{R}^{d}} \mathcal{F} \varphi(t, \cdot)(\xi) \overline{\mathcal{F} \phi(s, \cdot)(\xi)}|t-s|^{2 H-2} \mu(d \xi) d s d t
\end{aligned}
$$

where $\mathcal{F} \varphi$ refers to the Fourier transform with respect to the space variable only and the last equality in (2) follows from the simple properties of the Fourier transform. The map $\varphi \mapsto W(\varphi)$ defined in $\mathcal{D}\left([0, T] \times \mathbb{R}^{d}\right)$ can be extended to an isometry between $\mathcal{H}$ and the Gaussian space $H^{W}$ of $W$. For any $\varphi \in \mathcal{H}$, we denote this isometry by

$$
\varphi \mapsto W(\varphi)=\int_{0}^{T} \int_{\mathbb{R}^{d}} \varphi(t, x) W(d t, d x)
$$

Moreover, we can interchange the order of the integrals $d s d t$ and $\mu(d \xi)$, since for the indicator functions $\phi$ and $\varphi$, the integrand is a product of a function of $(t, s)$. Hence, we have

$$
\langle\varphi, \phi\rangle_{\mathcal{H}}=\alpha_{H} \int_{\mathbb{R}^{d}} \int_{0}^{T} \int_{0}^{T} \mathcal{F} \varphi(t, \cdot)(\xi) \overline{\mathcal{F} \phi(s, \cdot)(\xi)}|t-s|^{2 H-2} d s d t \mu(d \xi) .
$$

The space $\mathcal{H}$ may contain distributions, but it contains the space $|\mathcal{H}|$ of measurable functions $\varphi:[0, T] \mapsto \mathbb{R}^{d}$ such that

$$
\langle\varphi, \phi\rangle_{|\mathcal{H}|}=\alpha_{H} \int_{0}^{T} \int_{0}^{T} \int_{\mathbb{R}^{2 d}}|\varphi(t, x)||\phi(s, y)||t-s|^{2 H-2} \Lambda(x-y) d x d y d s d t<\infty .
$$


We shall make a standard assumption on the spectral measure $\mu$, which will prevail until the end of the paper (see Dalang [10] for some details about this hypothesis).

Hypothesis 1 The measure $\mu$ satisfies the following integrability condition:

$$
\int_{\mathbb{R}^{d}} \frac{1}{1+|\xi|^{2}} \mu(d \xi)<\infty
$$

Remark 2.1 Since the spectral measure $\mu$ is a non-trivial positive tempered measure, we can ensure that there exist positive constants $c_{1}, c_{2}$ and $k$ such that

$$
c_{1}<\int_{\{|\xi|<k\}} \mu(d \xi)<c_{2}
$$

The following estimate (see, for example, [18]) will be needed in the sequel: If $\frac{1}{2}<H<1$ and $f, g \in L^{\frac{1}{H}}([a, b])$, then

$$
\int_{a}^{b} \int_{a}^{b} f(x) g(y)|x-y|^{2 H-2} d x d y \leq C_{H}\|f\|_{L \frac{1}{H}([a, b])}\|g\|_{L^{\frac{1}{H}([a, b])}}
$$

where $C_{H}>0$ is a constant depending only on Hurst parameter $H$.

\subsection{Malliavin calculus}

Since $W=\left\{W(t, x),(t, x) \in[0, T] \times \mathbb{R}^{d}\right\}$ is Gaussian, we might develop the Malliavin calculus (refer to Nualart [18] for more details) with respect to fractional noise introduced in Sect. 2.1 in order to study the density of the solution to the SFKE (1). We will also recall briefly the results in Nourdin and Viens [17] in order to establish the lower and upper bounds for the density.

Recall the notation $W(\varphi)=\int_{0}^{T} \int_{\mathbb{R}^{d}} \varphi(t, x) W(d t, d x)$ for $\varphi \in \mathcal{H}$, and let $\mathcal{S}$ be the class of smooth and cylindrical random variables of the form

$$
F=f\left(W\left(\varphi_{1}\right), \ldots, W\left(\varphi_{n}\right)\right)
$$

where $f \in C_{b}^{\infty}\left(\mathbb{R}^{n}\right)$ (the set of all functions with bounded derivatives of all orders) and $\varphi_{i} \in \mathcal{H}(i=1, \ldots, n$ and $n \in \mathbb{N})$. For each $F \in \mathcal{S}$, define the derivative $D_{t, x} F$ by

$$
D_{t, x} F:=\sum_{i=1}^{n} \frac{\partial f}{\partial x_{i}}\left(W\left(\varphi_{1}\right), \ldots, W\left(\varphi_{n}\right)\right) \varphi_{i}(t, x)
$$

Let $\mathbb{D}^{1,2}$ be the completion of $\mathcal{S}$ under the norm

$$
\|F\|_{1,2}^{2}=\mathbb{E}\left[|F|^{2}+\|D F\|_{\mathcal{H}}^{2}\right] .
$$

Then $\mathbb{D}^{1,2}$ is the domain of the closed operator $D$ on $L^{2}(\Omega)$. We also denote by $\mathbb{D}_{h}$ the closure of $\mathcal{S}$ under the norm

$$
\|F\|_{h}^{2}=\mathbb{E}\left[|F|^{2}+\left|D_{h} F\right|^{2}\right]
$$


with $D_{h} F=\langle D F, h\rangle_{\mathcal{H}}$. Let $\left\{h_{h}, n \geq 1\right\}$ be an orthonormal basis of $\mathcal{H}$. Then $F \in \mathbb{D}^{1,2}$ if and only if $F \in \mathbb{D}_{h_{n}}$ for each $n \in \mathbb{N}$ and $\sum_{n=1}^{\infty} \mathbb{E}\left|D_{h_{n}} F\right|^{2}<\infty$.

On the other hand, the divergence operator $\delta$ is the adjoint of the derivative operator $D$ characterized by

$$
\mathbb{E}\langle D F, u\rangle_{\mathcal{H}}=\mathbb{E}(F \delta(u)), \quad \text { for any } F \in \mathcal{S},
$$

with $u \in L^{2}(\Omega ; \mathcal{H})$. Then $\operatorname{Dom}(\delta)$, the domain of $\delta$, is the set of all functions $u \in L^{2}(\Omega ; \mathcal{H})$ such that

$$
\mathbb{E}\left|\langle D F, u\rangle_{\mathcal{H}}\right| \leq C_{u}\|F\|_{\mathcal{H}},
$$

for all $F \in \mathbb{D}^{1,2}$, where $C_{u}$ is some constant depending on $u$.

Proposition 2.1 Let $\mathscr{F}_{A}:=\sigma\{W(B), B \subset A\}$ for $A \in \mathcal{B}\left([0, T] \times \mathbb{R}^{d}\right)$. If $F$ is a square integrable random variable that is measurable with respect to the $\sigma$-field $\mathscr{F}_{A^{c}}$, then

$$
D F 1_{A}=0 \quad \text { a.s. }
$$

Proposition 2.2 Let $F \in \mathbb{D}^{1,2}$. Then the distribution of the random variable $F$ is absolutely continuous with respect to Lebesgue measure if $\|D F\|_{\mathcal{H}}^{2}>0$ a.s.

We remark the following results.

\section{Remark 2.2}

1. Let $u=\left\{u(t, x),(t, x) \in[0, T] \times \mathbb{R}^{d}\right\}$ be an $\left\{\mathscr{F}_{t}, t \in[0, T]\right\}$-adapted random field. By Proposition 2.1, we have $D_{s, y} u(t, x)=0$, a.s. for any $0 \leq s<t \leq T$ and $x, y \in \mathbb{R}^{d}$.

2. Proposition 2.1 and Proposition 2.2 can be proved similarly to the work of Nualart [18] with Wiener white noise replaced by fractional noise introduced in

Sect. 2.2.

Another important operator in the theory of Malliavin calculus is the generator of the Ornstein-Uhlenbeck semigroup, which is usually denoted by $L$ (see, for example, Nualart [18]). It is related to the Malliavin derivative $D$ and its adjoint $\delta$ through the formula $\delta D F=-L F$ in the sense that $F$ belongs to the domain of $L$ if and only if it belongs to the domain of $\delta D$.

The authors in [17] considered a random variable $F \in \mathbb{D}^{1,2}$ with mean zero and defined the following function on $\mathbb{R}$ :

$$
g_{F}(z)=\mathbb{E}\left(\left\langle D F,-D L^{-1} F\right\rangle_{\mathcal{H}} \mid F=z\right)
$$

where $L^{-1}$ denotes the pseudo-inverse of $L$. Then Nourdin and Viens [17] proved the following.

Proposition 2.3 Assume that there exists a positive constant $C_{1}$, such that $g_{F}(F) \geq C_{1}>0$, a.s., then the law of $F$ has a density $p(\cdot)$ whose support is $\mathbb{R}$ and satisfies, almost everywhere 
in $\mathbb{R}$,

$$
p(z)=\frac{\mathbb{E}|F|}{2 g_{F}(z)} \exp \left\{-\int_{0}^{z} \frac{y}{g_{F}(y)} d y\right\}
$$

An immediate consequence of the above proposition, is that, if one also has $g_{F}(F) \leq C_{2}$ a.s., then the density $p(\cdot)$ satisfies, for almost all $z \in \mathbb{R}$,

$$
\frac{\mathbb{E}|F|}{2 C_{2}} \exp \left\{-\frac{z^{2}}{2 C_{1}}\right\} \leq p(z) \leq \frac{\mathbb{E}|F|}{2 C_{1}} \exp \left\{-\frac{z^{2}}{2 C_{2}}\right\}
$$

In order to deal with particular applications of this method, Proposition 3.7 in Nourdin and Viens [17] established an alternative formula for $g_{F}(F)$. That is,

$$
g_{F}(F)=\int_{0}^{+\infty} e^{-\eta} \mathbb{E}\left[\mathbb{E}^{\prime}\left(\langle D F, \widetilde{D F}\rangle_{\mathcal{H}} \mid F\right)\right] d \eta
$$

where, for any random variable $F$ defined in $(\Omega, \mathcal{F}, P), \widetilde{F}$ denotes the shifted random variable in $\Omega \times \Omega^{\prime}$, for some probability space $\Omega^{\prime}$, given by

$$
\widetilde{F}\left(\omega, \omega^{\prime}\right)=F\left(e^{-\zeta} \omega+\sqrt{1-e^{-2 \zeta}} \omega^{\prime}\right), \quad \omega \in \Omega, \omega^{\prime} \in \Omega^{\prime} .
$$

Notice that, indeed, $\widetilde{F}$ depends on the parameter $\zeta$, but we have decided to drop its explicit dependence for the sake of simplification. In Eq. (7), $\mathbb{E}^{\prime}$ stands for the expectation with respect to $\Omega^{\prime}$.

\section{Existence and uniqueness}

In this section, we will study the Cauchy problem for the SFKE (1) driven by fractional noise. Following Walsh [22], let us recall the notation of a mild solution to the SFKE (1).

Definition 3.1 An $L^{p}(\Omega) \mathscr{F}_{t}$-adapted process $u:[0, T] \times \mathbb{R}^{d} \times \Omega \rightarrow \mathbb{R}$ is a mild solution to the SFKE (1) if

$$
u(t, x)=\int_{0}^{t} \int_{\mathbb{R}^{d}} G(t-s, x-y) b(u(s, y)) d y d s+\int_{0}^{t} \int_{\mathbb{R}^{d}} G(t-s, x-y) W(d s, d y),
$$

where $G(t, x)$ is the fundamental solution (called also the Green function) of

$$
\frac{\partial}{\partial t} G(t, x)+(I-\Delta)^{\frac{\alpha}{2}}(-\Delta)^{\frac{\gamma}{2}} G(t, x)=0 .
$$

Moreover, according to [15], the Green function $G(t, x)$ can be written as

$$
G(t, x)=\frac{1}{(2 \pi)^{d}} \int_{\mathbb{R}^{d}} e^{\mathrm{i}\langle x, \xi\rangle} \exp \left\{-t|\xi|^{\alpha}\left(1+|\xi|^{2}\right)^{\frac{\gamma}{2}}\right\} d \xi,
$$

with $\mathrm{i}^{2}=-1$ and its Fourier transform $\mathcal{F} G(t, \cdot)(\xi)$ is given by

$$
\mathcal{F} G(t, \cdot)(\xi)=\exp \left\{-t|\xi|^{\alpha}\left(1+|\xi|^{2}\right)^{\frac{\gamma}{2}}\right\}
$$


When $H>1 / 2$, it turns out that under relatively mild assumptions on the fundamental solution $G$ given by (9), the condition

$$
\alpha_{H} \int_{0}^{T} \int_{0}^{T} \int_{\mathbb{R}^{d}} \mathcal{F} G(u, \cdot)(\xi) \overline{\mathcal{F} G(v, \cdot)(\xi)}|u-v|^{2 H-2} \mu(d \xi) d u d v<\infty
$$

provides a necessary and sufficient condition for the stochastic integral with respect to Gaussian process $W$ given by $\int_{0}^{t} \int_{\mathbb{R}^{d}} G(t-s, x-y) W(d s, d y)$ to be well-defined in $L^{p}(\Omega)$ with $p \geq 1$. Hence the condition (11) is also the necessary and sufficient condition for the existence of the solution in the linear case, i.e.

$$
\frac{\partial}{\partial t} u(t, x)+(I-\Delta)^{\frac{\alpha}{2}}(-\Delta)^{\frac{\gamma}{2}} u(t, x)=\dot{W}(t, x) .
$$

Next we firstly give an integrability condition on the spectral measure $\mu$.

Hypothesis 2 The measure $\mu$ satisfies the following integrability condition:

$$
\int_{\mathbb{R}^{d}}\left(\frac{1}{1+|\xi|^{2}}\right)^{H(\alpha+\gamma)} \mu(d \xi)<\infty .
$$

Before we prove the equivalence between (11) and (12). Let us now recall some of the main examples of spatially covariances $\Lambda(\cdot)$ (or the tempered measure $\mu$ ), which will be our guiding examples in the remainder of the present paper.

\section{Example 3.1}

1. Let $\Lambda(x)=\prod_{i=1}^{d} \alpha_{H_{i}}\left|x_{i}\right|^{2 H_{i}-2}$ be the covariance function of a fractional Brownian sheets with $H_{i}>\frac{1}{2}$ and $i=1, \ldots, d$. Then $\mu(d \xi)=\prod_{i=1}^{d} \alpha_{H_{i}}\left|\xi_{i}\right|^{-2 H_{i}+1} d \xi$. Then (12) is equivalent to $\sum_{i=1}^{d}\left(2 H_{i}-1\right)>d-2 H(\alpha+\gamma)$.

2. Let $\Lambda(x)=\gamma_{\lambda, d}|x|^{-(d-\lambda)}$ be the Riesz kernel of order $\lambda \in(0, d)$ with the constant $\gamma_{\lambda, d}$, then $\mu(d \xi)=|\xi|^{-\lambda} d \xi$ and the condition (12) is equivalent to $2 H(\alpha+\gamma)+\lambda>d$.

3. Let $\Lambda(x)=\gamma_{\lambda} \int_{0}^{\infty} \omega^{\frac{\lambda-d}{2}-1} e^{-\omega} e^{-\frac{|x|^{2}}{4 \omega}} d \omega$ be the Bessel kernel of order $\lambda>0$ with the constant $\gamma_{\lambda}$. Then $\mu(d \xi)=\left(1+|\xi|^{2}\right)^{-\frac{\lambda}{2}} d \xi$ the condition (12) is equivalent to $2 H(\alpha+\gamma)+\lambda>d$.

4. Let $\Lambda(0)<\infty$ (i.e. $\mu$ is a finite measure). It corresponds to a spatially smooth noise $\dot{W}$.

5. Suppose $d=1$ and $\Lambda=\delta_{0}$ (i.e. $\mu$ is the Lebesgue measure). This corresponds to a (rougher) noise $\dot{W}$ which is "white in space".

Proposition 3.1 Assume that the condition (12) holds, then (11) is satisfied.

Proof The proof of this proposition can be completed by using Proposition 3.2.

\section{Proposition 3.2 Denote}

$$
N_{t}(\xi)=\int_{0}^{t} \int_{0}^{t} \mathcal{F} G(u, \cdot)(\xi) \overline{\mathcal{F} G(v, \cdot)(\xi)}|u-v|^{2 H-2} d u d v .
$$

Then we have

$$
C_{3.1}\left(\frac{1}{1+|\xi|^{2}}\right)^{H(\alpha+\gamma)} \leq N_{t}(\xi) \leq C_{3.2}\left(\frac{1}{1+|\xi|^{2}}\right)^{H(\alpha+\gamma)},
$$


with two positive constants $C_{3.1}$ and $C_{3.2}$ given by $C_{3.1}=\frac{1}{4} \alpha_{H}^{-1}\left(t^{2 H}+2^{-2 H}\right)$ and $C_{3.2}=$ $\alpha_{H}^{-1} t^{2 H} 2^{H(\alpha+\gamma)}+C_{H} H^{2 H} 2^{H(\alpha+\gamma)}$ with $t \in[0, T]$.

Proof Recall that the Fourier transform $\mathcal{F G}(t, \cdot)(\xi)$ of $G(t, x)$ with respect to the spatial variable $x$ is given by (10). Then we can rewrite $N_{t}(\xi)$ defined by (13) as follows:

$$
N_{t}(\xi)=\int_{0}^{t} \int_{0}^{t} e^{-(u+v)|\xi|^{\alpha}\left(1+|\xi|^{2}\right)^{\frac{\gamma}{2}}}|u-v|^{2 H-2} d u d v .
$$

Now we want to find the lower and upper bound for $N_{t}(\xi)$. Firstly assuming that $|\xi|<1$ and using the fact $e^{-x} \leq 1$ for any $x>0$, then one obtains

$$
N_{t}(\xi) \leq \alpha_{H}^{-1} t^{2 H} \leq \alpha_{H}^{-1} t^{2 H} 2^{H(\alpha+\gamma)}\left(\frac{1}{1+|\xi|^{2}}\right)^{H(\alpha+\gamma)},
$$

where we have used the fact that $1<\frac{2}{1+|\xi|^{2}}$ when $|\xi|<1$.

Suppose now that $|\xi| \geq 1$, by means of the change of variables, inequality (5) and the fact that $1-e^{-x}<1$ for all $x>0$, we have

$$
\begin{aligned}
& \int_{0}^{t} \int_{0}^{t} e^{-(u+v)|\xi|^{\alpha}\left(1+|\xi|^{2}\right)^{\gamma / 2}}|u-v|^{2 H-2} d u d v \\
& \quad=\frac{1}{\left(|\xi|^{\alpha}\left(1+|\xi|^{2}\right)^{\gamma / 2}\right)^{2 H}} \int_{0}^{t|\xi|^{\alpha}\left(1+|\xi|^{2}\right)^{\gamma / 2}} \int_{0}^{t|\xi|^{\alpha}\left(1+|\xi|^{2}\right)^{\gamma / 2}} e^{-u-\nu}|u-v|^{2 H-2} d u d v \\
& \leq C_{H} \frac{H^{2 H}}{\left(|\xi|^{\alpha}\left(1+|\xi|^{2}\right)^{\gamma / 2}\right)^{2 H}}\left(1-e^{-\frac{t|\xi|^{\alpha}\left(1+|\xi|^{2} \gamma^{\gamma / 2}\right.}{H}}\right)^{2 H} \\
& \leq C_{H} H^{2 H} \frac{1}{|\xi|^{2 H(\alpha+\gamma)}} \\
& \leq C_{H} H^{2 H} 2^{H(\alpha+\gamma)}\left(\frac{1}{1+|\xi|^{2}}\right)^{H(\alpha+\gamma)}
\end{aligned}
$$

where we have used the fact $\frac{1}{|\xi|^{2 H(\alpha+\gamma)}} \leq\left(\frac{2}{1+|\xi|^{2}}\right)^{H(\alpha+\gamma)}$ if $|\xi| \geq 1$.

So combining the two estimates for $N_{t}(\xi)$ with $|\xi|<1$ and $|\xi| \geq 1$, we have

$$
N_{t}(\xi) \leq C_{3.2}\left(\frac{1}{1+|\xi|^{2}}\right)^{H(\alpha+\gamma)}
$$

with $C_{3.2}=\alpha_{H}^{-1} t^{2 H} 2^{H(\alpha+\gamma)}+C_{H} H^{2 H} 2^{H(\alpha+\gamma)}$.

Next let us proceed to prove the lower bound for $N_{t}(\xi)$. Suppose firstly $t|\xi|^{\alpha}\left(1+|\xi|^{2}\right)^{\frac{\gamma}{2}} \leq$ $\frac{1}{2}$, then, for any $u \in[0, t]$,

$$
u|\xi|^{\alpha}\left(1+|\xi|^{2}\right)^{\frac{\gamma}{2}} \leq t|\xi|^{\alpha}\left(1+|\xi|^{2}\right)^{\frac{\gamma}{2}} \leq \frac{1}{2} .
$$

Using $e^{-x}>1-x$ for any $x>0$, we conclude that

$$
\begin{aligned}
e^{-u|\xi|^{\alpha}\left(1+|\xi|^{2}\right)^{\frac{\gamma}{2}}} & \geq 1-u|\xi|^{\alpha}\left(1+|\xi|^{2}\right)^{\frac{\gamma}{2}} \\
& \geq 1-t|\xi|^{\alpha}\left(1+|\xi|^{2}\right)^{\frac{\gamma}{2}}
\end{aligned}
$$




$$
\geq \frac{1}{2}
$$

Hence one obtains that

$$
\begin{aligned}
N_{t}(\xi) & =\int_{0}^{t} \int_{0}^{t} e^{-(u+v)|\xi|^{\alpha}\left(1+|\xi|^{2}\right)^{\frac{\gamma}{2}}}|u-v|^{2 H-2} d u d \nu \\
& \geq \frac{1}{4} \int_{0}^{t} \int_{0}^{t}|u-v|^{2 H-2} d u d \nu \\
& \geq \frac{1}{4} \alpha_{H}^{-1} t^{2 H}\left(\frac{1}{1+|\xi|^{2}}\right)^{H(\alpha+\gamma)},
\end{aligned}
$$

where for the last inequality we have used the fact that $1 \geq \frac{1}{1+|\xi|^{2}}$.

Suppose now $t|\xi|^{\alpha}\left(1+|\xi|^{2}\right)^{\frac{\gamma}{2}} \geq \frac{1}{2}$, using the change of variables $u^{\prime}=u|\xi|^{\alpha}\left(1+|\xi|^{2}\right)^{\frac{\gamma}{2}}$ and $v^{\prime}=v|\xi|^{\alpha}\left(1+|\xi|^{2}\right)^{\frac{\gamma}{2}}$ and the fact $1-e^{-x}<x$ for all $x>0$, we get

$$
\begin{aligned}
& \int_{0}^{t} \int_{0}^{t} e^{-(u+v)|\xi|^{\alpha}\left(1+|\xi|^{2}\right)^{\gamma / 2}}|u-v|^{2 H-2} d u d v \\
& \quad=\frac{1}{\left(|\xi|^{\alpha}\left(1+|\xi|^{2}\right)^{\gamma / 2}\right)^{2 H}} \int_{0}^{t|\xi|^{\alpha}\left(1+|\xi|^{2}\right)^{\gamma / 2}} \int_{0}^{t|\xi|^{\alpha}\left(1+|\xi|^{2}\right)^{\gamma / 2}} e^{-u-v}|u-v|^{2 H-2} d u d v \\
& \quad \geq \frac{1}{\left(|\xi|^{\alpha}\left(1+|\xi|^{2}\right)^{\gamma / 2}\right)^{2 H}} \int_{0}^{1 / 2} \int_{0}^{1 / 2} e^{-u-v}|u-v|^{2 H-2} d u d v \\
& \geq \frac{1}{4} \frac{1}{\left(|\xi|^{\alpha}\left(1+|\xi|^{2}\right)^{\gamma / 2}\right)^{2 H}} \int_{0}^{1 / 2} \int_{0}^{1 / 2}|u-v|^{2 H-2} d u d v \\
& \geq \frac{1}{\alpha_{H} 2^{2+2 H}}\left(\frac{1}{1+|\xi|^{2}}\right)^{H(\alpha+\gamma)}
\end{aligned}
$$

where we have used the fact that $|\xi|^{\alpha}\left(1+|\xi|^{2}\right)^{\frac{\gamma}{2}} \leq\left(1+|\xi|^{2}\right)^{\frac{\alpha+\gamma}{2}}$. Thus we can conclude to the following lower bound for $N_{t}(\xi)$ :

$$
N_{t}(\xi) \geq C_{3.1}\left(\frac{1}{1+|\xi|^{2}}\right)^{H(\alpha+\gamma)}
$$

with $C_{3.1}=\frac{1}{4} \alpha_{H}^{-1}\left(t^{2 H}+2^{-2 H}\right)$. Thus the proof of this proposition is complete.

Remark 3.1 Note that Proposition 3.1 extends the case of stochastic heat equation in Balan and Tudor [8] with condition (12) (i.e. $\alpha=0$ and $\gamma=2$ ). Moreover, Proposition 3.1 is also an improvement of Lemma 2.1 obtained in [15] and also generalizes the cases in MárquezCarreras [16] (with $\alpha=0$ and $\gamma>0$ ). Moreover, we also prove the equivalence between (11) and (12) in this paper.

Now we can state the main result in this section. The proof of this theorem could be derived by the standard arguments with some estimates of the Green function $G(t, x)$ and some properties of the stochastic integral in (8). However, we have preferred to give the complete proof. We shall also make the following hypothesis on the coefficient $b$. 
(H.1): The function $b$ satisfies the Lipschitz condition as follows:

$$
|b(x)-b(y)| \leq C|x-y|, \quad \forall x, y \in \mathbb{R}^{d} .
$$

Theorem 3.1 Assume that (12) holds and the coefficient $b$ satisfies (H.1), then there exists a unique solution $u(t, x)$ to the SFKE (1) such that

$$
\sup _{(t, x) \in[0, T] \times \mathbb{R}^{d}} \mathbb{E}|u(t, x)|^{p}<\infty
$$

for any $T>0$ and $p \geq 2$.

Firstly let us give a useful estimate associated with the Green function $G(t, x)$ given by (9).

Lemma 3.1 We have the following, with $t \in[0, T]$ :

$$
\int_{\mathbb{R}^{d}} G(t, x)^{2} d x \asymp t^{-\frac{d}{\alpha+\gamma}}
$$

where the notation $f \asymp g$ means that there exist two constants $c, C$ such that $c g \leq f \leq C g$.

Proof Using the Plancherel theorem and equality (10), we can write

$$
\begin{aligned}
\int_{\mathbb{R}^{d}} G(t, x)^{2} d x & =\frac{1}{(2 \pi)^{d}} \int_{\mathbb{R}^{d}}|\mathcal{F} G(t, \cdot)(\xi)|^{2} d \xi \\
& =\frac{1}{(2 \pi)^{d}} \int_{\mathbb{R}^{d}} \exp \left\{-2 t|\xi|^{\alpha}\left(1+|\xi|^{2}\right)^{\gamma / 2}\right\} d \xi \\
& =\frac{S_{d}}{(2 \pi)^{d}} \int_{0}^{+\infty} r^{d-1} \exp \left\{-2 \operatorname{tr}^{\alpha}\left(1+r^{2}\right)^{\gamma / 2}\right\} d r
\end{aligned}
$$

where we have used the integration in polar coordinates in the last equation above and $S_{d}$ is a positive constant resulting from the integration over the angular spherical coordinates. Now using the fact $r^{\alpha}\left(1+r^{2}\right)^{\gamma / 2} \geq r^{\alpha+\gamma}$ with $r>0$, we get, with the change of variable formula $u=2 t r^{\alpha+\gamma}$,

$$
\begin{aligned}
\int_{0}^{+\infty} r^{d-1} \exp \left\{-2 t r^{\alpha}\left(1+r^{2}\right)^{\gamma / 2}\right\} d r & \leq \int_{0}^{+\infty} r^{d-1} \exp \left\{-2 t r^{\alpha+\gamma}\right\} d r \\
& =(2 t)^{-\frac{d}{\alpha+\gamma}} \frac{1}{\alpha+\gamma} \int_{0}^{+\infty} u^{\frac{d}{\alpha+\gamma}-1} e^{-u} d u \\
& =(2 t)^{-\frac{d}{\alpha+\gamma}} \frac{1}{\alpha+\gamma} \Gamma\left(\frac{d}{\alpha+\gamma}\right) t^{-\frac{d}{\alpha+\gamma}}
\end{aligned}
$$

where $\Gamma\left(\frac{d}{\alpha+\gamma}\right)$ is a Gamma function.

On the other hand, using the fact $r^{\alpha}\left(1+r^{2}\right)^{\gamma / 2} \leq\left(1+r^{2}\right)^{\frac{\alpha+\gamma}{2}}$ with $r>0$, we get with the change of variable formula $u=2 t\left(1+r^{2}\right)^{\frac{\alpha+\gamma}{2}}$,

$$
\int_{0}^{+\infty} r^{d-1} \exp \left\{-2 t r^{\alpha}\left(1+r^{2}\right)^{\gamma / 2}\right\} d r
$$




$$
\begin{aligned}
& \geq \int_{0}^{+\infty} r^{d-1} \exp \left\{-2 t\left(1+r^{2}\right)^{\frac{\alpha+\gamma}{2}}\right\} d r \\
& =\frac{1}{2}(2 t)^{-\frac{2}{\alpha+\gamma}} \int_{0}^{+\infty}\left(\left(\frac{u}{2 t}\right)^{\frac{2}{\alpha+\gamma}}-1\right)^{\frac{d}{2}-1} u^{\frac{2}{\alpha+\gamma}-1} e^{-u} d u \\
& \approx \frac{1}{2}(2 t)^{-\frac{d}{\alpha+\gamma}} \int_{0}^{+\infty} u^{\frac{d}{\alpha+\gamma}-1} e^{-u} d u, \quad \text { as } u \rightarrow \infty,
\end{aligned}
$$

where the last integral is finite. Then we can conclude the proof of this lemma.

Now let us prove the main result in this section.

Proof of Theorem 3.1 We use the Picard approximation to get a solution to (8). Define

$$
\begin{aligned}
u^{(n+1)}(t, x)= & \int_{0}^{t} \int_{\mathbb{R}^{d}} G(t-s, x-y) b\left(u^{(n)}(s, y)\right) d y d s \\
& +\int_{0}^{t} \int_{\mathbb{R}^{d}} G(t-s, x-y) W(d s, d y) .
\end{aligned}
$$

Firstly, we will prove that

$$
\sup _{n \in \mathbb{N} \cup\{0\}} \sup _{(t, x) \in[0, T] \times \mathbb{R}^{d}} \mathbb{E}\left|u^{(n)}(t, x)\right|^{p}<\infty
$$

It follows from (17) that for each $n \in \mathbb{N}$

$$
\mathbb{E}\left|u^{(n+1)}(t, x)\right|^{p} \leq C_{p}\left(A_{p}^{(n)}(t, x)+B_{p}^{(n)}(t, x)\right)
$$

where

$$
A_{p}^{(n)}(t, x)=\mathbb{E}\left|\int_{0}^{t} \int_{\mathbb{R}^{d}} G(t-s, x-y) b\left(u^{(n)}(s, y)\right) d y d s\right|^{p}
$$

and

$$
B_{p}^{(n)}(t, x)=\mathbb{E}\left|\int_{0}^{t} \int_{\mathbb{R}^{d}} G(t-s, x-y) W(d s, d y)\right|^{p}
$$

Note that, by the Hölder inequality and the fact that $G(t-s, x-y)$ is $\mathbb{R}^{d}$-integrable for $t \neq s$,

$$
\begin{aligned}
A_{p}^{(n)}(t, x) \leq & C_{p}\left(\int_{0}^{t} \int_{\mathbb{R}^{d}}|G(t-s, x-y)| d y d s\right)^{p-1} \\
& \times \mathbb{E}\left(\int_{0}^{t} \int_{\mathbb{R}^{d}}\left|b\left(u^{(n)}(s, y)\right)\right|^{p} \cdot|G(t-s, x-y)| d y d s\right) \\
\leq & C_{p} \mathbb{E}\left[\int_{0}^{t} \int_{\mathbb{R}^{d}}\left(1+\left|u^{(n)}(s, y)\right|^{p}\right) \cdot|G(t-s, x-y)| d y d s\right] \\
\leq & C_{p} \int_{0}^{t}\left(1+\sup _{y \in \mathbb{R}^{d}} \mathbb{E}\left|u^{(n)}(s, y)\right|^{p}\right)\left(\int_{\mathbb{R}^{d}}|G(t-s, x-y)| d y\right) d s .
\end{aligned}
$$


For the term $B_{p}^{(n)}(t, x)$, since the stochastic integral $\int_{0}^{t} \int_{\mathbb{R}^{d}} G(t-s, x-y) W(d s, d y)$ is Gaussian, according to Proposition 3.2 and Eq. (12), we have

$$
\begin{aligned}
B_{p}^{(n)}(t, x) & =\mathbb{E}\left|\int_{0}^{t} \int_{\mathbb{R}^{d}} G(t-s, x-y) W(d s, d y)\right|^{p} \\
& \leq C_{p, H}\left(\int_{0}^{t} \int_{0}^{t} \int_{\mathbb{R}^{d}} \mathcal{F} G(u, \cdot)(\xi) \overline{\mathcal{F} G(v, \cdot)(\xi)}|u-v|^{2 H-2} \mu(d \xi) d u d v\right)^{\frac{p}{2}} \\
& \leq C_{p, T, H} \int_{\mathbb{R}^{d}}\left(\frac{1}{1+|\xi|^{2}}\right)^{H(\alpha+\gamma)} \mu(d \xi)<\infty .
\end{aligned}
$$

Combining (18), (19) and (20), we have

$$
\sup _{x \in \mathbb{R}^{d}} \mathbb{E}\left|u^{(n+1)}(t, x)\right|^{p} \leq C_{p} \int_{0}^{t}\left(1+\sup _{y \in \mathbb{R}^{d}} \mathbb{E}\left|u^{(n)}(s, y)\right|^{p}\right) \int_{\mathbb{R}^{d}}|G(t-s, x-y)| d y d s .
$$

Then that fact that $|G(t-s, x-y)|$ is $\mathbb{R}^{d}$-integrable for $t \neq s$ in (19) together with the Gronwall lemma ensures that

$$
\sup _{0 \leq t \leq T} \sup _{x \in \mathbb{R}^{d}} \mathbb{E}\left|u^{(n+1)}(t, x)\right|^{p}<\infty
$$

and consequently $\left\{u^{(n)}(t, x), n \geq 1\right\}$ is well defined. Moreover, by Lemma 15 in Dalang [10], one can obtain

$$
\sup _{n \in \mathbb{N} \cup\{0\}} \sup _{(t, x) \in[0, T] \times \mathbb{R}^{d}} \mathbb{E}\left|u^{(n)}(t, x)\right|^{p}<\infty
$$

Secondly let us prove that $\left\{u^{(n)}(t, x), n \geq 1\right\}$ converges in $L^{p}(\Omega)$. As for $n \geq 2$,

$$
\begin{aligned}
\mathbb{E} & \left(\left|u^{(n+1)}(t, x)-u^{(n)}(t, x)\right|^{p}\right) \\
& =\mathbb{E}\left(\left|\int_{0}^{t} \int_{\mathbb{R}^{d}} G(t-s, x-y)\left[b\left(u^{(n)}(s, y)\right)-b\left(u^{(n-1)}(s, y)\right)\right] d y d s\right|^{p}\right) \\
& \leq C_{p} \int_{0}^{t} \int_{\mathbb{R}^{d}} \mathbb{E}\left|u^{(n)}(s, y)-u^{(n-1)}(s, y)\right|^{p}|G(t-s, x-y)| d y d s \\
& \leq C_{p} \int_{0}^{t} \sup _{y \in \mathbb{R}^{d}} \mathbb{E}\left|u^{(n)}(s, y)-u^{(n-1)}(s, y)\right|^{p}\left(\int_{\mathbb{R}^{d}}|G(t-s, x-y)| d y\right) d s .
\end{aligned}
$$

Then Gronwall's lemma yields

$$
\sum_{n \geq 0} \sup _{(t, x) \in[0, T] \times \mathbb{R}^{d}} \mathbb{E}\left(\left|u^{(n+1)}(t, x)-u^{(n)}(t, x)\right|^{p}\right)<\infty .
$$

Hence, $\left\{u^{(n)}(t, x)\right\}_{n \geq 0}$ is a Cauchy sequence in $L^{p}(\Omega)$. Let

$$
u(t, x)=\lim _{n \rightarrow \infty} u^{(n)}(t, x) .
$$


Then, for each $(t, x) \in[0, T] \times \mathbb{R}^{d}$,

$$
\sup _{(t, x) \in[0, T] \times \mathbb{R}^{d}} \mathbb{E}|u(t, x)|^{p}<\infty
$$

Taking $n \rightarrow \infty$ in $L^{p}(\Omega)$ in both sides of (17) shows that $u=\left\{u(t, x) ;(t, x) \in[0, T] \times \mathbb{R}^{d}\right\}$ satisfies (3.1).

The uniqueness can be checked by a standard argument.

\section{Remark 3.2}

1. Our result Theorem 3.1 here is an extension of the one in Márquez-Carreras [15] (when $H=1 / 2$ ) to fractional noise. However, the noises considered in Márquez-Carreras [15] is multiplicative.

2. The cases $\alpha=0$ and $\gamma=2$, then Eq. (1) reduces to the classical stochastic heat equation with fractional version considered in Balan and Tudor [8]. Then the condition (12) is coherent with the results found in [8] for the stochastic heat equation with fractional noise.

\section{Index- $\beta$ Gaussian random field}

In this section we will prove that the solution to the SFKE (1) satisfies the following property defined by Definition 4.1; see Márquez-Carreras [15] for example. As a related problem, we also study the sample paths of the solution to the SFKE (1).

Definition 4.1 Let $X(t)$ be a Gaussian process that has zero mean, stationary increments, and a continuous covariance function. Set $\sigma^{2}(\theta)=\mathbb{E}\left[|X(t+\theta)-X(t)|^{2}\right]$. Then, if there exists $\beta \in(0,1]$ such that

$$
\beta=\sup \left\{\widetilde{\beta}: \sigma(\theta)=o\left(\|\theta\|^{\widetilde{\beta}}\right),\|\theta\| \rightarrow 0\right\}
$$

then $X=\left\{X(t), t \in \mathbb{R}^{d}\right\}$ is called an index- $\beta$ Gaussian field.

Example 4.1 From the above definition of index- $\beta$ Gaussian random fields, it is the local variance of their increments which determines the degree of fractality of the sample path. For example, from the covariance function of fractional Brownian motion $B^{H}=\left\{B^{H}(t), t \in\right.$ $[0, T]\}$,

$$
\mathbb{E}\left(B^{H}(t) B^{H}(s)\right)=\frac{1}{2}\left(t^{2 H}+s^{2 H}-|t-s|^{2 H}\right),
$$

it is seen that $B^{H}$ is an index- $H$ random field.

Moreover, from Angulo et al. [1], the following results hold with probability one.

1. $\operatorname{dim}_{H}(\operatorname{graph}(X))=d+1-\beta$, where $\operatorname{dim}_{H}$ is the Hausdorff dimension which quantifies the irregularity of a set and $\operatorname{graph}(X):=\left\{(t, X(t)), t \in \mathbb{R}^{d}\right\}$.

2. $X$ is Hölder continuous of order $\rho$ strictly less than $\beta$. However, for any $\rho<\beta$, $X$ fails to satisfy any uniform Hölder condition of order $\rho$.

In this section we essentially show that the solution to the SFKE (1) has similar properties to the solution studied in Angulo et al. [1], Márquez-Carreras [15]. However before we 
state our main result in this section, we will firstly give another condition on the tempered measure $\mu$ which is slightly stronger than Hypothesis 2 because of the appearance of $\eta \in$ $(0,1)$.

Hypothesis 3 For some $\psi \in(0,1)$, the measure $\mu$ satisfies the following integrability condition:

$$
\int_{\mathbb{R}^{d}}\left(\frac{1}{1+|\xi|^{2}}\right)^{H(\alpha+\gamma) \psi} \mu(d \xi)<\infty
$$

Let us denote

$$
U(t, x)=\int_{0}^{t} \int_{\mathbb{R}^{d}} G(t-s, x-y) W(d s, d y)
$$

which is the mild solution to the SFKE (1) with $b=0$. Then we have

Theorem 4.1 Assume that the spectral measure $\mu$ satisfies (24). Then, for a fixed $t \in[0, T]$ and $x, x^{\prime} \in \mathbb{R}^{d}$, the spatial covariance function of $U(t, x)$ given by $(25)$ is

$$
R_{t}\left(x-x^{\prime}\right)=\int_{\mathbb{R}^{d}} e^{-\mathrm{i}\left\langle\xi \xi x-x^{\prime}\right\rangle} \frac{\left\|e^{-u}\right\|_{\mathcal{H}\left(\left[0, t|\xi|^{\alpha}\left(1+|\xi|^{2}\right)^{\gamma / 2}\right]\right)}^{2}}{\left(|\xi|^{\alpha}\left(1+|\xi|^{2}\right)^{\gamma / 2}\right)^{2 H}} \mu(d \xi) .
$$

Moreover, at each fixed time $t \in[0, T]$,

- if $H(\alpha+\gamma)>1$ then $U(t, \cdot)$ is an index- $\beta_{1}$ Gaussian field with $\beta_{1} \in(0,1)$;

- if $H(\alpha+\gamma) \leq 1$ then $U(t, \cdot)$ is an index- $\beta_{2}$ Gaussian field with $\beta_{2} \in(0, H(\alpha+\gamma)(1-\psi))$ for $\psi \in(0,1)$.

Proof We first calculate the spatial covariance function for a fixed $t \in[0, T]$. By means of the definition of the Fourier transform, a change of variable formula and Fubini's theorem, we obtain, for any $x, x^{\prime} \in \mathbb{R}^{d}$,

$$
\begin{aligned}
\mathbb{E} & \left(U(t, x) U\left(t, x^{\prime}\right)\right) \\
& =\int_{0}^{t} \int_{0}^{t} \int_{\mathbb{R}^{d}} \mathcal{F} G\left(t-s_{1}, x-\cdot\right)(\xi) \cdot \overline{\mathcal{F} G\left(t-s_{2}, x^{\prime}-\cdot\right)(\xi) \mid}\left|s_{1}-s_{2}\right|^{2 H-2} \mu(d \xi) d s_{1} d s_{2} \\
& =\int_{\mathbb{R}^{d}} \int_{0}^{t} \int_{0}^{t} e^{-\mathrm{i}(\xi, x\rangle} \mathcal{F} G\left(t-s_{1}, \cdot\right)(\xi) \cdot \overline{e^{-\mathrm{i} \xi \cdot x^{\prime}} \mathcal{F} G\left(t-s_{2}, \cdot\right)(\xi)}\left|s_{1}-s_{2}\right|^{2 H-2} d s_{1} d s_{2} \mu(d \xi) \\
& =\int_{\mathbb{R}^{d}} e^{-\mathrm{i}\left(\xi, x-x^{\prime}\right\rangle} \int_{0}^{t} \int_{0}^{t} e^{-\left(2 t-s_{1}-s_{2}\right)|\xi|^{\alpha}\left(1+|\xi|^{2}\right)^{\gamma / 2}}\left|s_{1}-s_{2}\right|^{2 H-2} d s_{1} d s_{2} \mu(d \xi) \\
& =\int_{\mathbb{R}^{d}} e^{-\mathrm{i}\left(\xi, x-x^{\prime}\right\rangle} \frac{\left\|e^{-u}\right\|_{\mathcal{H}\left(\left[0, t|\xi|^{\alpha}\left(1+|\xi|^{2}\right)^{\gamma / 2}\right]\right)}^{2}}{\left(|\xi|^{\alpha}\left(1+|\xi|^{2}\right)^{\gamma / 2}\right)^{2 H}}(d \xi),
\end{aligned}
$$

where we use the notation $\left\|e^{-u}\right\|_{\mathcal{H}\left(\left[0, t|\xi|^{\alpha}\left(1+|\xi|^{2}\right)^{\gamma / 2}\right]\right)}^{2}$ to denote the double integral

$$
\int_{0}^{t|\xi|^{\alpha}\left(1+|\xi|^{2}\right)^{\gamma / 2}} \int_{0}^{t|\xi|^{\alpha}\left(1+|\xi|^{2}\right)^{\gamma / 2}} e^{-u_{1}-u_{2}}\left|u_{1}-u_{2}\right|^{2 H-2} d u_{1} d u_{2}
$$


which is finite. In fact using the change of variables formula $u_{1}=\left(t-s_{1}\right)|\xi|^{\alpha}\left(1+|\xi|^{2}\right)^{\gamma / 2}$ and $u_{2}=\left(t-s_{2}\right)|\xi|^{\alpha}\left(1+|\xi|^{2}\right)^{\gamma / 2}$, then we have

$$
\begin{aligned}
& \int_{0}^{t} \int_{0}^{t} e^{-\left(2 t-s_{1}-s_{2}\right)|\xi|^{\alpha}\left(1+|\xi|^{2}\right)^{\gamma / 2}}\left|s_{1}-s_{2}\right|^{2 H-2} d s_{1} d s_{2} \\
& \quad=\frac{1}{\left(|\xi|^{\alpha}\left(1+|\xi|^{2}\right)^{\gamma / 2}\right)^{2 H}} \int_{0}^{t|\xi|^{\alpha}\left(1+|\xi|^{2}\right)^{\gamma / 2}} \int_{0}^{t|\xi|^{\alpha}\left(1+|\xi|^{2}\right)^{\gamma / 2}} e^{-u_{1}-u_{2}}\left|u_{1}-u_{2}\right|^{2 H-2} d u_{1} d u_{2} \\
& \quad \leq \frac{1}{\left(|\xi|^{\alpha}\left(1+|\xi|^{2}\right)^{\gamma / 2}\right)^{2 H}} \alpha_{H}^{-1}\left(t|\xi|^{\alpha}\left(1+|\xi|^{2}\right)^{\gamma / 2}\right)^{2 H} \\
& \quad=\alpha_{H}^{-1} t^{2 H}
\end{aligned}
$$

Moreover, for a fixed time $t \in[0, T]$, the process $U(t, x)$ is a Gaussian field that has zero mean, stationary increments and a continuous covariance function.

We now begin to study the index. For $t \in[0, T], x \in \mathbb{R}^{d}$ and small $\theta \in \mathbb{R}^{d}$, we have

$$
\begin{aligned}
\sigma^{2}(\theta)= & \mathbb{E}|U(t, x+\theta)-U(t, x)|^{2} \\
= & \mathbb{E}\left|\int_{0}^{t} \int_{\mathbb{R}^{d}}(G(t-s, x+\theta-y)-G(t-s, x-y)) W(d s, d y)\right|^{2} \\
= & \int_{0}^{t} \int_{0}^{t} \int_{\mathbb{R}^{d}} \mathcal{F}\left(G\left(t-s_{1}, x+\theta-\cdot\right)-G\left(t-s_{1}, x-\cdot\right)\right)(\xi) \\
& \times \overline{\mathcal{F}\left(G\left(t-s_{2}, x+\theta-\cdot\right)-G\left(t-s_{2}, x-\cdot\right)\right)(\xi)}\left|s_{1}-s_{2}\right|^{2 H-2} \mu(d \xi) d s_{1} d s_{2} .
\end{aligned}
$$

Then using the formula

$$
\begin{aligned}
\mathcal{F} & (G(t-s, x+\theta-\cdot)-G(t-s, x-\cdot))(\xi) \\
& =e^{-\mathrm{i}\langle\xi,(x+\theta)\rangle} \mathcal{F}(G(t-s, \cdot))(\xi)-e^{-\mathrm{i}\langle\xi, x\rangle} \mathcal{F}(G(t-s, \cdot))(\xi) \\
& =e^{-\mathrm{i}\langle\xi, x\rangle}\left[e^{-\mathrm{i}\langle\xi, \theta\rangle}-1\right] \mathcal{F}(G(t-s, \cdot))(\xi) .
\end{aligned}
$$

Then we can rewrite (26) as follows:

$$
\begin{aligned}
\sigma^{2}(\theta)= & \int_{0}^{t} \int_{0}^{t} \int_{\mathbb{R}^{d}} \mathcal{F}\left(G\left(t-s_{1}, x+\theta-\cdot\right)-G\left(t-s_{1}, x-\cdot\right)\right)(\xi) \\
& \times \overline{\mathcal{F}\left(G\left(t-s_{2}, x+\theta-\cdot\right)-G\left(t-s_{2}, x-\cdot\right)\right)(\xi)}\left|s_{1}-s_{2}\right|^{2 H-2} \mu(d \xi) d s_{1} d s_{2} \\
= & \int_{0}^{t} \int_{0}^{t} \int_{\mathbb{R}^{d}}\left|e^{-\mathrm{i}(\xi, x)}\right|^{2}\left|1-e^{-\mathrm{i}\langle\xi, \theta\rangle}\right|^{2} \mathcal{F}\left(G\left(t-s_{1}, \cdot\right)\right)(\xi) \overline{\mathcal{F}\left(G\left(t-s_{2}, \cdot\right)\right)(\xi)} \\
& \times\left|s_{1}-s_{2}\right|^{2 H-2} \mu(d \xi) d s_{1} d s_{2} \\
= & \int_{0}^{t} \int_{0}^{t} \int_{\mathbb{R}^{d}}\left|1-e^{-\mathrm{i}(\xi, \theta\rangle}\right|^{2} \mathcal{F}\left(G\left(t-s_{1}, \cdot\right)\right)(\xi) \\
& \times \overline{\mathcal{F}\left(G\left(t-s_{2}, \cdot\right)\right)(\xi)}\left|s_{1}-s_{2}\right|^{2 H-2} \mu(d \xi) d s_{1} d s_{2} \\
= & \int_{0}^{t} \int_{0}^{t} \int_{\mathbb{R}^{d}}\left|1-e^{-\mathrm{i}\langle\xi, \theta\rangle}\right|^{2} e^{-\left(2 t-s_{1}-s_{2}\right)|\xi|^{\alpha}\left(1+|\xi|^{2}\right)^{\gamma / 2}}\left|s_{1}-s_{2}\right|^{2 H-2} \mu(d \xi) d s_{1} d s_{2} .
\end{aligned}
$$


Using the fact $\mathbb{R}^{d}=\left\{|\xi|^{2}<1\right\} \cup\left\{|\xi|^{2} \geq 1\right\}$, we can divide $\sigma^{2}(\theta)$ into two parts $\sigma^{2}(\theta) 1_{\left\{|\xi|^{2}<1\right\}}$ and $\sigma^{2}(\theta) 1_{\left\{|\xi|^{2} \geq 1\right\}}$ according to the value of $|\xi|$. Let us firstly estimate the term $\sigma^{2}(\theta) 1_{\left\{|\xi|^{2}<1\right\}}$. Recall the following inequality:

$$
\left|1-e^{-\mathrm{i}\langle\xi, \theta\rangle}\right|^{2}=4 \sin ^{2}\left(\frac{\langle\xi, \theta\rangle}{2}\right) \leq|\xi|^{2}|\theta|^{2} .
$$

Then with (4) in Remark 2.1, we have

$$
\begin{aligned}
\sigma^{2}(\theta) 1_{\left\{|\xi|^{2}<1\right\}} & \leq|\theta|^{2} \int_{\left\{|\xi|^{2}<1\right\}}|\xi|^{2} \int_{0}^{t} \int_{0}^{t}\left|s_{1}-s_{2}\right|^{2 H-2} d s_{1} d s_{2} \mu(d \xi) \\
& \leq \alpha_{H}^{-1}|\theta|^{2} t^{2 H} \int_{\left\{|\xi|^{2}<1\right\}} \mu(d \xi) \\
& \leq C|\theta|^{2} .
\end{aligned}
$$

For the proof of the second term $\sigma^{2}(\theta) 1_{\left\{|\xi|^{2}>1\right\}}$, we distinguish two cases depending on the value of $H(\alpha+\gamma)$. We first study the case $H(\alpha+\gamma)>1$. With inequality (28), we have

$$
\begin{aligned}
\sigma^{2}(\theta) 1_{\left\{|\xi|^{2}>1\right\}} & \leq \alpha_{H}^{-1} t^{2 H}|\theta|^{2} \int_{|\xi|>1} \frac{|\xi|^{2}}{\left(|\xi|^{\alpha}\left(1+|\xi|^{2}\right)^{\gamma / 2}\right)^{2 H}} \mu(d \xi) \\
& \leq \alpha_{H}^{-1} t^{2 H}|\theta|^{2} \int_{|\xi|>1} \frac{1}{|\xi|^{2(H(\alpha+\gamma)-1)}} \mu(d \xi) \\
& \leq \alpha_{H}^{-1} 2^{H(\alpha+\gamma)-1} t^{2 H}|\theta|^{2} \int_{|\xi|>1}\left(\frac{1}{1+|\xi|^{2}}\right)^{H(\alpha+\gamma)-1} \mu(d \xi) .
\end{aligned}
$$

Next, according to Hypothesis 3, we just need choose a constant $\psi$ satisfying $0<\psi<$ $1-\frac{1}{H(\alpha+\gamma)}$, then we can conclude that

$$
\int_{|\xi|>1}\left(\frac{1}{1+|\xi|^{2}}\right)^{H(\alpha+\gamma)-1} \mu(d \xi) \leq \int_{|\xi|>1}\left(\frac{1}{1+|\xi|^{2}}\right)^{H(\alpha+\gamma) \psi} \mu(d \xi)<\infty .
$$

This yields

$$
\begin{aligned}
\sigma^{2}(\theta) 1_{\left\{|\xi|^{2}>1\right\}} & \leq \alpha_{H}^{-1} 2^{H(\alpha+\gamma)-1} t^{2 H} \int_{|\xi|>1}\left(\frac{1}{1+|\xi|^{2}}\right)^{H(\alpha+\gamma)-1} \mu(d \xi)|\theta|^{2} \\
& =C_{4.1}|\theta|^{2}
\end{aligned}
$$

with $C_{4.1}=\alpha_{H}^{-1} 2^{H(\alpha+\gamma)-1} t^{2 H} \int_{|\xi|>1}\left(\frac{1}{1+|\xi|^{2}}\right)^{H(\alpha+\gamma)-1} \mu(d \xi)$.

For the case $H(\alpha+\gamma) \leq 1$, we need the following inequality, with $0<\kappa<1$ :

$$
\left|1-e^{-\mathrm{i}\langle\xi, \theta\rangle}\right|^{2} \leq 4^{1-\kappa}|\xi|^{2 \kappa}|\theta|^{2 \kappa}
$$

Then one gets

$$
\sigma^{2}(\theta) 1_{\left\{|\xi|^{2}>1\right\}} \leq \alpha_{H}^{-1} t^{2 H} \int_{|\xi|>1}\left|1-e^{-\mathrm{i}\{\xi, \theta\rangle}\right|^{2}\left(\frac{1}{|\xi|^{\alpha}\left(1+|\xi|^{2}\right)^{\gamma / 2}}\right)^{2 H} \mu(d \xi)
$$




$$
\begin{aligned}
& \leq 4^{1-\kappa} \alpha_{H}^{-1} t^{2 H}|\theta|^{2 \kappa} \int_{|\xi|>1} \frac{1}{|\xi|^{2(H(\alpha+\gamma)-\kappa)}} \mu(d \xi) \\
& \leq 4^{1-\kappa} \alpha_{H}^{-1} t^{2 H}|\theta|^{2 \kappa} \int_{|\xi|>1}\left(\frac{1}{1+|\xi|^{2}}\right)^{H(\alpha+\gamma)-\kappa} \mu(d \xi) \\
& \leq 4^{1-\kappa} \alpha_{H}^{-1} t^{2 H}|\theta|^{2 \kappa} \int_{|\xi|>1}\left(\frac{1}{1+|\xi|^{2}}\right)^{H(\alpha+\gamma) \psi} \mu(d \xi),
\end{aligned}
$$

provided we choose a positive constant $\kappa$ satisfying $0<\kappa<H(\alpha+\gamma)(1-\psi)$. Then we conclude that

$$
\begin{aligned}
\sigma^{2}(\theta) 1_{\left\{|\xi|^{2}>1\right\}} & \leq 4^{1-\kappa} \alpha_{H}^{-1} t^{2 H}|\theta|^{2 \kappa} \int_{|\xi|>1}\left(\frac{1}{1+|\xi|^{2}}\right)^{H(\alpha+\gamma) \psi} \mu(d \xi) \\
& =C_{4.2}|\theta|^{2 \kappa}, \quad 0<\kappa<H(\alpha+\gamma)(1-\psi) .
\end{aligned}
$$

Combining the above estimates (29), (30), (31) and (33) for $\sigma^{2}(\theta)$, we conclude that

$$
\sigma^{2}(\theta) \leq \begin{cases}C|\theta|^{2 \beta} & \text { if } H(\alpha+\gamma)>1, \\ C|\theta|^{2 \kappa} & \text { if } H(\alpha+\gamma) \leq 1\end{cases}
$$

with $\beta \in(0,1), \psi \in(0,1)$ and $0<\kappa<H(\alpha+\gamma)(1-\psi)$.

Next let us move to the case of $U$ with respect to the time variable. The result is given as follows.

Theorem 4.2 Assume that the measure $\mu$ satisfies Hypothesis 3 for some $\eta \in(0,1)$. Then, for $t \in \mathbb{R}_{+}, \tau \in \mathbb{R}$ such that $t+\tau \in \mathbb{R}_{+}$and $x, z \in \mathbb{R}^{d}$, the spatial-temporal covariance function of $U(t+\tau, x)$ and $U(t, z)$ is

$$
\begin{aligned}
R_{t}(\tau, x-z)= & \int_{\mathbb{R}^{d}} e^{-\mathrm{i}(\xi, x-z)} e^{-\tau|\xi|^{\alpha}\left(1+|\xi|^{2}\right)^{\gamma / 2}} \\
& \times \int_{0}^{t+\tau} \int_{0}^{t} e^{-\left(2 t-s_{1}-s_{2}\right)|\xi|^{\alpha}\left(1+|\xi|^{2}\right)^{\gamma / 2}}\left|s_{1}-s_{2}\right|^{2 H-2} d s_{1} d s_{2} \mu(d \xi) .
\end{aligned}
$$

Moreover, $U(\cdot, x)$ is asymptotically in time with an index- $\beta$ Gaussian field with $0<\beta<$ $H(1-\psi)$.

Since the process $U$ is not stationary in time but as $t$ tends to infinity, it converges to a stationary process. That means the limiting-time process is stationary in time and space.

Proof For $t \in[0, T]$ and $\tau \in \mathbb{R}$ such that $t+\tau \in[0, T]$ and $x, z \in \mathbb{R}^{d}$, with Fubini's theorem,

$$
\begin{aligned}
& \mathbb{E}(U(t+\tau, x) \overline{U(t, z)}) \\
& \quad=\int_{0}^{t+\tau} \int_{0}^{t} \int_{\mathbb{R}^{d}} \mathcal{F} G\left(t+\tau-s_{1}, x-\cdot\right)(\xi) \overline{\mathcal{F} G\left(t-s_{2}, z-\cdot\right)(\xi)}\left|s_{1}-s_{2}\right|^{2 H-2} \mu(d \xi) d s_{1} d s_{2} \\
& \quad=\int_{0}^{t+\tau} \int_{0}^{t} \int_{\mathbb{R}^{d}} e^{-\mathrm{i}\langle\xi, x-z\rangle} \mathcal{F} G\left(t+\tau-s_{1}, \cdot\right)(\xi) \overline{\mathcal{F} G\left(t-s_{2}, \cdot\right)(\xi)}\left|s_{1}-s_{2}\right|^{2 H-2} \mu(d \xi) d s_{1} d s_{2}
\end{aligned}
$$




$$
=\int_{\mathbb{R}^{d}} e^{-\mathrm{i}\langle\xi, x-z\rangle} \int_{0}^{t+\tau} \int_{0}^{t} e^{-\left(t+\tau-s_{1}+t-s_{2}\right)|\xi|^{\alpha}\left(1+|\xi|^{2}\right)^{\gamma / 2}}\left|s_{1}-s_{2}\right|^{2 H-2} d s_{1} d s_{2} \mu(d \xi)
$$

In the following we should prove that the integral $\int_{0}^{t+\tau} \int_{0}^{t} e^{-\left(t+\tau-s_{1}+t-s_{2}\right)|\xi|^{\alpha}\left(1+|\xi|^{2}\right)^{\gamma / 2}} \mid s_{1}-$ $\left.s_{2}\right|^{2 H-2} d s_{1} d s_{2}$ is finite. Actually with (5) one gets

$$
\begin{aligned}
\int_{0}^{t+\tau} & \int_{0}^{t} e^{-\left(t+\tau-s_{1}+t-s_{2}\right)|\xi|^{\alpha}\left(1+|\xi|^{2}\right)^{\gamma / 2}\left|s_{1}-s_{2}\right|^{2 H-2} d s_{1} d s_{2}} \\
\leq & C_{H}\left(\int_{0}^{t+\tau} \exp \left\{-\frac{\left(t+\tau-s_{1}\right)|\xi|^{\alpha}\left(1+|\xi|^{2}\right)^{\gamma / 2}}{H}\right\} d s_{1}\right)^{H} \\
& \times\left(\int_{0}^{t} \exp \left\{-\frac{\left(t-s_{2}\right)|\xi|^{\alpha}\left(1+|\xi|^{2}\right)^{\gamma / 2}}{H}\right\} d s_{1}\right)^{H} \\
= & C_{H}\left(\frac{H}{|\xi|^{\alpha}\left(1+|\xi|^{2}\right)^{\gamma / 2}}\right)^{2 H}\left[1-e^{-\left(t+\tau \frac{|\xi|^{\alpha}\left(1+|\xi|^{2}\right)^{\gamma / 2}}{H}\right.}\right]\left[1-e^{-\frac{|\xi|^{\alpha}\left(1+|\xi|^{2}\right)^{\gamma / 2}}{H}}\right] .
\end{aligned}
$$

On the other hand, one gets

$$
\begin{aligned}
\int_{0}^{t+\tau} & \int_{0}^{t} e^{-\left(t+\tau-s_{1}+t-s_{2}\right)|\xi|^{\alpha}\left(1+|\xi|^{2}\right)^{\gamma / 2}}\left|s_{1}-s_{2}\right|^{2 H-2} d s_{1} d s_{2} \\
= & \int_{0}^{t} \int_{0}^{t} e^{-\left(t+\tau-s_{1}+t-s_{2}\right)|\xi|^{\alpha}\left(1+|\xi|^{2}\right)^{\gamma / 2}}\left|s_{1}-s_{2}\right|^{2 H-2} d s_{1} d s_{2} \\
& \quad+\int_{t}^{t+\tau} \int_{0}^{t} e^{-\left(t+\tau-s_{1}+t-s_{2}\right)|\xi|^{\alpha}\left(1+|\xi|^{2}\right)^{\gamma / 2}}\left|s_{1}-s_{2}\right|^{2 H-2} d s_{1} d s_{2} \\
& :=I_{1}+I_{2} .
\end{aligned}
$$

With the change of variables formula and the definition of the norm in $\mathcal{H}$, one can get

$$
\begin{aligned}
I_{1} & =\left(\frac{1}{|\xi|^{\alpha}\left(1+|\xi|^{2}\right)^{\gamma / 2}}\right)^{2 H} e^{-\tau|\xi|^{\alpha}\left(1+|\xi|^{2}\right)^{\gamma / 2}}\left\|e^{-u}\right\|_{\mathcal{H}\left(\left[0, t|\xi|^{\alpha}\left(1+|\xi|^{2}\right)^{\gamma / 2}\right]\right)} \\
& \rightarrow \frac{\Gamma(2 H)}{2 H-1}\left(\frac{1}{|\xi|^{\alpha}\left(1+|\xi|^{2}\right)^{\gamma / 2}}\right)^{2 H} e^{-\tau|\xi|^{\alpha}\left(1+|\xi|^{2}\right)^{\gamma / 2}} \text { as } t \rightarrow+\infty
\end{aligned}
$$

where $\Gamma(\cdot)$ is the gamma function. For the second term $I_{2}$, one can show that it tends to zero as $t \rightarrow \infty$. Thus we complete the proof of (35).

We now tackle the second part of this theorem. We assume that $x \in \mathbb{R}^{d}, t \in[0, T]$ and $\tau \in \mathbb{R}$ such that $t+\tau \in[0, T]$. Then we have

$$
\begin{aligned}
\mathbb{E}|U(t+\tau, x)-U(t, x)|^{2} \\
=\mathbb{E}\left|\int_{0}^{t+\tau} \int_{\mathbb{R}^{d}} G(t+\tau-s, x-y) W(d s, d y)-\int_{0}^{t} \int_{\mathbb{R}^{d}} G(t-s, x-y) W(d s, d y)\right|^{2} \\
\leq 2\left(\mathbb{E}\left|\int_{t}^{t+\tau} \int_{\mathbb{R}^{d}} G(t+\tau-s, x-y) W(d s, d y)\right|^{2}\right. \\
\left.\quad+\mathbb{E}\left|\int_{0}^{t} \int_{\mathbb{R}^{d}}(G(t+\tau-s, x-y)-G(t-s, x-y)) W(d s, d y)\right|^{2}\right) \\
:=2\left(A_{1}+A_{2}\right) .
\end{aligned}
$$


We firstly study the term $A_{1}$. In fact according to the inner product (2) and Fourier transform for $G$ we have

$$
\begin{aligned}
& A_{1}=\mathbb{E}\left|\int_{t}^{t+\tau} \int_{\mathbb{R}^{d}} G(t+\tau-s, x-y) W(d s, d y)\right|^{2} \\
& =\int_{t}^{t+\tau} \int_{t}^{t+\tau} \int_{\mathbb{R}^{d}} \mathcal{F} G\left(t+\tau-s_{1}, x-\cdot\right)(\xi) \\
& \times \overline{\mathcal{F} G\left(t+\tau-s_{2}, x-\cdot\right)(\xi)}\left|s_{1}-s_{2}\right|^{2 H-2} \mu(d \xi) d s_{1} d s_{2} \\
& =\int_{\mathbb{R}^{d}} \int_{t}^{t+\tau} \int_{t}^{t+\tau} e^{-\left(2(t+\tau)-s_{1}-s_{2}\right)|\xi|^{\alpha}\left(1+|\xi|^{2}\right)^{\gamma / 2}}\left|s_{1}-s_{2}\right|^{2 H-2} d s_{1} d s_{2} \mu(d \xi) \\
& =\int_{|\xi| \leq 1} \int_{t}^{t+\tau} \int_{t}^{t+\tau} e^{-\left(2(t+\tau)-s_{1}-s_{2}\right)|\xi|^{\alpha}\left(1+|\xi|^{2}\right)^{\gamma / 2}}\left|s_{1}-s_{2}\right|^{2 H-2} d s_{1} d s_{2} \mu(d \xi) \\
& +\int_{|\xi|>1} \int_{t}^{t+\tau} \int_{t}^{t+\tau} e^{-\left(2(t+\tau)-s_{1}-s_{2}\right)|\xi|^{\alpha}\left(1+|\xi|^{2}\right)^{\gamma / 2}}\left|s_{1}-s_{2}\right|^{2 H-2} d s_{1} d s_{2} \mu(d \xi) \\
& :=A_{1,1}+A_{1,2} \text {. }
\end{aligned}
$$

By means of the inequalities (5) and $1-e^{-x} \leq x$ for all $x>0$, we can prove that

$$
\begin{aligned}
A_{1,1} & \leq C_{H} \int_{|\xi| \leq 1}\left(\int_{t}^{t+\tau} e^{-\frac{(t+\tau-s)|\xi|^{\alpha}\left(1+|\xi|^{2}\right)^{\gamma / 2}}{H}} d s\right)^{2 H} \mu(d \xi) \\
& =C_{H} \int_{|\xi| \leq 1} \frac{H^{2 H}}{\left(|\xi|^{\alpha}\left(1+|\xi|^{2}\right)^{\gamma / 2}\right)^{2 H}}\left(1-e^{-\frac{\tau|\xi|^{\alpha}\left(1+|\xi|^{2} \gamma^{\gamma / 2}\right.}{H}}\right)^{2 H} \mu(d \xi) \\
& \leq C_{H} \int_{|\xi| \leq 1} \mu(d \xi)|\tau|^{2 H},
\end{aligned}
$$

and also with (5), by choosing some constant $\theta=1-\eta \in(0,1)$, we can prove that under Hypothesis 3

$$
\begin{aligned}
A_{1,2} & \leq C_{H} \int_{|\xi|>1}\left(\int_{t}^{t+\tau} e^{-\frac{(t+\tau-s)|\xi|^{\alpha}\left(1+|\xi|^{2}\right)^{\gamma / 2}}{H}} d s\right)^{2 H} \mu(d \xi) \\
& =C_{H} \int_{|\xi|>1} \frac{H^{2 H}}{\left(|\xi|^{\alpha}\left(1+|\xi|^{2}\right)^{\gamma / 2}\right)^{2 H}}\left(1-e^{-\frac{-\tau|\xi|^{\alpha}\left(1+|\xi|^{2} \gamma^{\gamma / 2}\right.}{H}}\right)^{2 H} \mu(d \xi) \\
& \leq C_{H} \int_{|\xi|>1} \frac{H^{2 H}}{\left(|\xi|^{\alpha}\left(1+|\xi|^{2}\right)^{\gamma / 2}\right)^{2 H}}\left(1-e^{-\frac{\tau|\xi|^{\alpha}\left(1+|\xi|^{2}\right)^{\gamma / 2}}{H}}\right)^{2 H \theta} \mu(d \xi) \\
& \leq C_{H} \tau^{2 H \theta} \int_{|\xi|>1} \frac{1}{|\xi|^{2 H(\alpha+\gamma)(1-\theta)}} \mu(d \xi) \\
& \leq 2^{H(\alpha+\gamma)(1-\theta)} C_{H} \tau^{2 H \theta} \int_{|\xi|>1}\left(\frac{1}{1+|\xi|^{2}}\right)^{2 H(\alpha+\gamma)(1-\theta)} \mu(d \xi) \\
& =C_{4.2}|\tau|^{2 H \theta}, \theta=1-\eta \in(0,1),
\end{aligned}
$$

with $t \in[0, T], \tau \in \mathbb{R}$ such that $t+\tau \in[0, T]$ and

$$
C_{4.2}=C_{H} 2^{H(\alpha+\gamma)(1-\theta)} \int_{|\xi|>1}\left(\frac{1}{1+|\xi|^{2}}\right)^{2 H(\alpha+\gamma)(1-\theta)} \mu(d \xi) .
$$


Combining (38), (39) and (40), we conclude that

$$
A_{1} \leq C_{4.3}|\tau|^{2 H \theta}, \quad \theta=1-\eta \in(0,1)
$$

with $C_{4.3}=C_{H} \int_{|\xi| \leq 1} \mu(d \xi)+C_{4.2}$. Finally let us study the second term, $A_{2}$,

$$
\begin{aligned}
& A_{2}=\mathbb{E}\left|\int_{0}^{t} \int_{\mathbb{R}^{d}}(G(t+\tau-s, x-y)-G(t-s, x-y)) W(d s, d y)\right|^{2} \\
& =\int_{0}^{t} \int_{0}^{t} \int_{\mathbb{R}^{d}} \mathcal{F}\left(G\left(t+\tau-s_{1}, x-\cdot\right)-G\left(t-s_{1}, x-\cdot\right)\right)(\xi) \\
& \times \overline{\mathcal{F}\left(G\left(t+\tau-s_{2}, x-\cdot\right)-G\left(t-s_{2}, x-\cdot\right)\right)(\xi)}\left|s_{1}-s_{2}\right|^{2 H_{2}} \mu(d \xi) d s_{1} d s_{2} \\
& =\int_{0}^{t} \int_{0}^{t} \int_{\mathbb{R}^{d}} e^{-\left(2 t-s_{1}-s_{2}\right)|\xi|^{\alpha}\left(1+|\xi|^{2}\right)^{\gamma / 2}}\left|1-e^{-\tau|\xi|^{\alpha}\left(1+|\xi|^{2}\right)^{\gamma / 2}}\right|^{2}\left|s_{1}-s_{2}\right|^{2 H-2} \mu(d \xi) d s_{1} d s_{2} \\
& =\int_{\mathbb{R}^{d}}\left|1-e^{-\tau|\xi|^{\alpha}\left(1+|\xi|^{2}\right)^{\gamma / 2}}\right|^{2} \int_{0}^{t} \int_{0}^{t} e^{-\left(2 t-s_{1}-s_{2}\right)|\xi|^{\alpha}\left(1+|\xi|^{2}\right)^{\gamma / 2}}\left|s_{1}-s_{2}\right|^{2 H-2} d s_{1} d s_{2} \mu(d \xi) \\
& =\int_{|\xi| \leq 1}\left|1-e^{-\tau|\xi|^{\alpha}\left(1+|\xi|^{2}\right)^{\gamma / 2}}\right|^{2} \int_{0}^{t} \int_{0}^{t} e^{-\left(2 t-s_{1}-s_{2}\right)|\xi|^{\alpha}\left(1+|\xi|^{2}\right)^{\gamma / 2}}\left|s_{1}-s_{2}\right|^{2 H-2} d s_{1} d s_{2} \mu(d \xi) \\
& +\int_{|\xi|>1}\left|1-e^{-\tau|\xi|^{\alpha}\left(1+|\xi|^{2}\right)^{\gamma / 2}}\right|^{2} \int_{0}^{t} \int_{0}^{t} e^{-\left(2 t-s_{1}-s_{2}\right)|\xi|^{\alpha}\left(1+|\xi|^{2}\right)^{\gamma / 2}} \\
& \times\left|s_{1}-s_{2}\right|^{2 H-2} d s_{1} d s_{2} \mu(d \xi) \\
& :=A_{2,1}+A_{2,2} \text {. }
\end{aligned}
$$

With similar calculations to (39) and the fact that $1-e^{-x} \leq x$ for all $x>0$, we can bound $A_{2,1}$ as follows with $\theta=1-\eta \in(0,1)$ :

$$
\begin{aligned}
A_{2,1} & \leq C_{H} t^{2 H} \int_{|\xi| \leq 1}\left|1-e^{-\tau|\xi|^{\alpha}\left(1+|\xi|^{2}\right)^{\gamma / 2}}\right|^{2 \theta} \mu(d \xi) \\
& \leq C_{H} t^{2 H} \int_{|\xi| \leq 1}\left(\tau|\xi|^{\alpha}\left(1+|\xi|^{2}\right)^{\gamma / 2}\right)^{2 \theta} \leq C_{4.4}|\tau|^{2 \theta} .
\end{aligned}
$$

Let $\beta \in(0, H(1-\eta))$ with $\eta \in(0,1)$. For the double integral $\int_{0}^{t} \int_{0}^{t} e^{-\left(2 t-s_{1}-s_{2}\right)|\xi|^{\alpha}\left(1+|\xi|^{2}\right)^{\gamma / 2}} \mid s_{1}-$ $\left.s_{2}\right|^{2 H-2} d s_{1} d s_{2}$ in $A_{2,2}$, by means of (5), we can bound it as follows:

$$
\begin{aligned}
& \int_{0}^{t} \int_{0}^{t} e^{-\left(2 t-s_{1}-s_{2}\right)|\xi|^{\alpha}\left(1+|\xi|^{2}\right)^{\gamma / 2}}\left|s_{1}-s_{2}\right|^{2 H-2} d s_{1} d s_{2} \\
& \quad=\frac{1}{\left(|\xi|^{\alpha}\left(1+|\xi|^{2}\right)^{\gamma / 2}\right)^{2 H}} \int_{0}^{t|\xi|^{\alpha}\left(1+|\xi|^{2}\right)^{\gamma / 2}} \int_{0}^{t|\xi|^{\alpha}\left(1+|\xi|^{2}\right)^{\gamma / 2}} e^{-u_{1}-u_{2}}\left|u_{1}-u_{2}\right|^{2 H-2} d u_{1} d u_{2} \\
& \quad \leq C_{H} \frac{H^{2 H}}{\left(|\xi|^{\alpha}\left(1+|\xi|^{2}\right)^{\gamma / 2}\right)^{2 H}}\left(1-e^{-\frac{t|\xi|^{\alpha}\left(1+\left.|\xi|^{2}\right|^{\gamma / 2}\right.}{H}}\right)^{2 H} \\
& \quad \leq C_{H} H^{2 H} \frac{1}{|\xi|^{2(\alpha+\gamma) H}} .
\end{aligned}
$$


Thus with the inequality $\left|1-e^{-x}\right| \leq 2^{1-\beta}\left|1-e^{-x}\right|^{\beta}$ for some $\beta \in(0, H(1-\eta)) \subset(0,1)$ and the fact $1-e^{-x} \leq x$ for all $x>0$, we have with Hypothesis 3

$$
\begin{aligned}
A_{2,2} & \leq C_{H} H^{2 H} \int_{|\xi|>1}\left|1-e^{-\tau|\xi|^{\alpha}\left(1+|\xi|^{2}\right)^{\gamma / 2}}\right|^{2} \frac{1}{|\xi|^{2(\alpha+\gamma) H}} \mu(d \xi) \\
& \leq C_{H} H^{2 H} 2^{2-2 \beta} \int_{|\xi|>1}\left|1-e^{-\tau|\xi|^{\alpha}\left(1+|\xi|^{2}\right)^{\gamma / 2}}\right|^{2 \beta}\left(\frac{1}{|\xi|^{(\alpha+\gamma)}}\right)^{2 H} \mu(d \xi) \\
& \leq C_{H} H^{2 H} 2^{2-2 \beta}|\tau|^{2 \beta} \int_{|\xi|>1}\left(\frac{1}{|\xi|^{(\alpha+\gamma)}}\right)^{2(H-\beta)} \mu(d \xi) \\
& \leq C_{H} H^{2 H} 2^{2-2 \beta+(H-\beta)(\alpha+\gamma)}|\tau|^{2 \beta} \int_{|\xi|>1}\left(\frac{1}{1+|\xi|^{2}}\right)^{(H-\beta)(\alpha+\gamma)} \mu(d \xi) \\
& \leq C_{4.6}|\tau|^{2 \beta}, 0<\beta<H(1-\eta),
\end{aligned}
$$

with $C_{4.6}=C_{H} H^{2 H} 2^{2-2 \beta+(H-\beta)(\alpha+\gamma)} \int_{|\xi|>1}\left(\frac{1}{1+|\xi|^{2}}\right)^{(H-\beta)(\alpha+\gamma)} \mu(d \xi)$. Putting together (37), (38), (39), (40), (41), (42) and (43), we then can conclude that with $\theta=1-\eta \in(0,1)$ and $\beta \in$ $(0, H(1-\eta))$

$$
\mathbb{E}|U(t+\tau, x)-U(t, x)|^{2} \leq C_{4.7}\left(|\tau|^{2 H \theta}+|\tau|^{2 \beta}\right) \leq C_{4.8}|\tau|^{2 \beta} .
$$

Finally in this section, as a related problem, we can also get the following path Hölder regularity of $U(t, x)$ with respect to the time and space variables, respectively, by following similar arguments to the proof of Theorem 4.1 and Theorem 4.2.

Proposition 4.1 Assume that the spectral measure $\mu$ satisfies Hypothesis 3 for some $\psi \in$ $(0,1)$. Then, for every $t, s \in[0, T], T>0, x, y \in \mathbb{R}^{d}, p \geq 2, \beta_{1} \in(0, H(1-\psi))$ and $\beta_{2} \in(0,1)$ and $\beta_{3} \in(0, H(\alpha+\gamma)(1-\psi))$, we have

$$
\mathbb{E}|U(t, x)-U(s, x)|^{p} \leq C_{4.9}|t-s|^{p \beta_{1}} ;
$$

and

$$
\mathbb{E}|U(t, x)-U(t, y)|^{p} \leq \begin{cases}C_{4.10}|x-y|^{p \beta_{2}} & \text { if } H(\alpha+\gamma)>1, \\ C_{4.11}|x-y|^{p \beta_{3}} & \text { if } H(\alpha+\gamma) \leq 1\end{cases}
$$

\section{Analysis of the density}

This section is devoted to a study of the density of the solution to the SFKE (1) at any fixed $(t, x) \in[0, T] \times \mathbb{R}^{d}$. This will be done by using Malliavin calculus. The aim in this section is two-fold. Firstly we will prove that the solution to the SFKE (1) at any fixed $(t, x) \in[0, T] \times \mathbb{R}^{d}$ is a random variable whose equation admits a density. Secondly we apply the results obtained by Nourdin and Viens [17] to the SFKE (1) to obtain the upper and lower Gaussian-type estimates for the density (see recent work by Nualart and QuerSardanyons [19, 20], and Liu and Yan [14]).

\subsection{Existence of the density}

The main result in this subsection is stated as follows. 
Theorem 5.1 We set the conditions of Theorem 3.1 and $d<\alpha+\gamma$, furthermore we also assume that $b(\cdot) \in C^{1}(\mathbb{R})$ with bounded Lipschitz continuous derivative. Then, for any fixed $(t, x) \in[0, T] \times \mathbb{R}^{d}$, the equation of the solution to Eq. (1) is absolutely continuous with respect to the Lebesgue measure.

Before giving the proof of Theorem 5.1, we firstly give the following.

Proposition 5.1 Assume that the spectral measure $\mu$ satisfies Hypothesis 2, suppose also that $d<\alpha+\gamma$ and the coefficient $b(\cdot)$ is $C^{1}(\mathbb{R})$ with bounded Lipschitz continuous derivative. Then, for any fixed $(t, x) \in[0, T] \times \mathbb{R}^{d}$, the random variable $u(t, x)$ belongs to $\mathbb{D}^{1,2}$ and satisfies

$$
D_{v, z} u(t, x)=G(t-v, x-z)+\int_{v}^{t} \int_{\mathbb{R}^{d}} G(t-s, x-y) b^{\prime}(u(s, y)) D_{v, z} u(s, y) d y d s,
$$

for all $0<v \leq t$ and $x \in \mathbb{R}^{d}$.

Proof Let $u^{(n)}(t, x)(n \geq 1)$ be the solution of Eq. (17). Since $b$ is Lipschitz, by a standard argument, one can see that the sequences $u^{(n)}$ converges to $u$ in $L^{p}(\Omega)$ for any $p \geq 2$ and $(t, x) \in[0, T] \times \mathbb{R}^{d}$ as $n \rightarrow \infty$. Then a similar argument to that in Zhang and Zheng [23] shows that, for each $n \in \mathbb{N}$ and $h \in \mathcal{H}, u^{(n)}(t, x) \in \mathbb{D}_{h}$,

$$
\begin{aligned}
D_{h} u^{(n)}(t, x)= & \int_{0}^{t} \int_{\mathbb{R}^{d}} G(t-s, x-y) b^{\prime}\left(u^{(n-1)}(s, y)\right) D_{h} u^{(n-1)}(s, y) d y d s \\
& +\langle G(t-\cdot, x-\cdot), h\rangle_{\mathcal{H}} .
\end{aligned}
$$

Since $u^{(n)}(t, x) \rightarrow u(t, x)$ as $n \rightarrow \infty$ in the $L^{p}(\Omega)$ sense, there exists a random field $u_{h}(t, x)$ such that $D_{h} u^{(n)}(t, x) \rightarrow u_{h}(t, x)$ as $n \rightarrow \infty$ uniformly on $(t, x) \in[0, T] \times \mathbb{R}^{d}$, and the latter satisfies

$$
u_{h}(t, x)=\int_{0}^{t} \int_{\mathbb{R}^{d}} G(t-s, x-y) b^{\prime}(u(s, y)) u_{h}(s, y) d y d s+\langle G(t-\cdot, x-\cdot), h\rangle_{\mathcal{H}} .
$$

Hence, from the closeness of the operator $D_{h}$, it follows that $u(t, x) \in \mathbb{D}_{h}, D_{h} u(t, x)=u_{h}(t, x)$ and

$$
D_{h} u(t, x)=\int_{0}^{t} \int_{\mathbb{R}^{d}} G(t-s, x-y) b^{\prime}(u(s, y)) D_{h} u(s, y) d y d s+\langle G(t-\cdot, x-\cdot), h\rangle_{\mathcal{H}} .
$$

Next we proceed to proving that $u(t, x) \in \mathbb{D}^{1,2}$. Recall the sequence $\left\{h_{n}, n \geq 1\right\}$ introduced in Sect. 2. By (48), one gets

$$
\begin{aligned}
\mathbb{E}\left|D_{h_{n}} u(t, x)\right|^{2} & \\
= & \mathbb{E} \mid \int_{0}^{t} \int_{\mathbb{R}^{d}} G(t-s, x-y) b^{\prime}(u(s, y)) D_{h_{n}} u(s, y) d y d s+\left\langle G(t-\cdot, x-\cdot),\left.\left.h_{n}\right|_{\mathcal{H}}\right|^{2}\right. \\
\leq & C_{5.1 .1} \mathbb{E}\left[\int_{0}^{t} \int_{\mathbb{R}^{d}}(G(t-s, x-y))^{2}\left(D_{h_{n}} u(s, y)\right)^{2} d y d s\right] \\
& +C_{5.1 .2}\left\langle G(t-\cdot, x-\cdot),\left.h_{n}\right|_{\mathcal{H}^{\prime}} ^{2},\right.
\end{aligned}
$$


with two positive constants $C_{5.1 .1}$ and $C_{5.1 .2}$. Set

$$
U_{m}(t)=\sup _{x \in \mathbb{R}^{d}} \mathbb{E} \sum_{n=1}^{m}\left|D_{h_{n}} u(t, x)\right|^{2}
$$

Then, by (49), the Hölder inequality with $p=q=2$ and estimates (16) for the Green function, we have

$$
\begin{aligned}
U_{m}(t) & \leq C_{5.1 .3} \mathbb{E}\left[\int_{0}^{t} \int_{\mathbb{R}^{d}}(G(t-s, x-y))^{2} U_{m}(s) d y d s\right]+C_{5.1 .4}\|G(t-\cdot, x-\cdot)\|_{\mathcal{H}}^{2} \\
& \leq C_{5.1 .5}+C_{5.1 .6} \int_{0}^{t}(t-s)^{-\frac{d}{\alpha+\gamma}} U_{m}(s) d s .
\end{aligned}
$$

Then the Gronwall lemma yields

$$
U_{m}(t) \leq C_{5.1 .7} \exp \left\{C_{5.1 .8} T^{1-\frac{d}{\alpha+\gamma}}\right\}
$$

where $C_{5.1 .7}$ and $C_{5.1 .8}$ are independent of $m$. Let $m \rightarrow \infty$ to get

$$
\sup _{x \in \mathbb{R}} \mathbb{E} \sum_{n=1}^{\infty}\left|D_{h_{n}} u(t, x)\right|^{2}<\infty
$$

That means that $u(t, x) \in \mathbb{D}^{1,2}$.

Since $u(t, x)$ is $\mathscr{F}_{t}$-adapted, there exists a measurable function $D_{v, z} u(t, x) \in \mathcal{H}$ such that $D_{v, z} u(t, x)=0$ if $v>t$ and for any $h \in \mathcal{H}$

$$
D_{h} u(t, x)=\langle D u(t, x), h\rangle_{\mathcal{H}} .
$$

From (48), (51) and Fubini's theorem, it follows that

$$
\begin{aligned}
\langle D u(t, x), h\rangle_{\mathcal{H}} \\
\quad=\int_{0}^{t} \int_{\mathbb{R}^{d}} G(t-s, x-y) b^{\prime}(u(s, y))\langle D u(s, y), h\rangle_{\mathcal{H}} d y d s+\langle G(t-\cdot, x-\cdot), h\rangle_{\mathcal{H}} \\
\quad=\left\langle\int_{0}^{t} \int_{\mathbb{R}^{d}} G(t-s, x-y) b^{\prime}(u(s, y)) D u(s, y) d y d s, h\right\rangle_{\mathcal{H}}+\langle G(t-\cdot, x-\cdot), h\rangle_{\mathcal{H}} .
\end{aligned}
$$

Therefore

$$
D_{v, z} u(t, x)=\int_{v}^{t} \int_{\mathbb{R}^{d}} G(t-s, x-y) b^{\prime}(u(s, y)) D_{v, z} u(s, y) d y d s+G(t-v, x-y) .
$$

Thus we can conclude the proof of this proposition.

We also need the following lemma concerning the estimates for the $L^{2}$-norm of the Malliavin derivative $D u(t, x)$. 
Lemma 5.1 For $\varepsilon \in(0, t)$ and $d<\alpha+\gamma$, there exist two positive constants $C_{5.1 .9}$ and $C_{5.1 .10}$ such that

$$
\sup _{s \in[t-\varepsilon, t]} \sup _{y \in \mathbb{R}^{d}} \mathbb{E}\left(\int_{t-\varepsilon}^{t} \int_{\mathbb{R}^{d}}\left|D_{v, z} u(s, y)\right|^{2} d z d v\right)<C_{5.1 .9} \varepsilon^{1-\frac{d}{\alpha+\gamma}}
$$

and

$$
\sup _{\zeta \in \mathbb{R}} \sup _{s \in[t-\varepsilon, t]} \sup _{y \in \mathbb{R}^{d}} \mathbb{E}\left(\int_{t-\varepsilon}^{t} \int_{\mathbb{R}^{d}} \mathbb{E}^{\prime}\left(\left|\widetilde{D_{v, z} \mathcal{u}(s, y)}\right|^{2}\right) d z d v\right)<C_{5.1 .10} \varepsilon^{1-\frac{d}{\alpha+\gamma}} .
$$

Proof We will only deal with the proof of (52), since (53) can be checked by using exactly the same arguments. For $s \in[t-\varepsilon, t]$, set

$$
L_{\varepsilon}(s, y)=\mathbb{E}\left(\int_{t-\varepsilon}^{s} \int_{\mathbb{R}^{d}}\left|D_{v, z} u(s, y)\right|^{2} d z d v\right) .
$$

Then from the proof of Proposition 5.1, we get

$$
\sup _{(s, y) \in[0, T] \times \mathbb{R}^{d}} L_{\varepsilon}(s, y)<\infty .
$$

Let us invoke the linear equation (45) satisfied by the Malliavin derivative $D u(s, y)$ for $(s, y) \in[t-\varepsilon, t] \times \mathbb{R}^{d}$, then

$$
\begin{aligned}
L_{\varepsilon}(s, y) \leq & 2\left[\int_{t-\varepsilon}^{s} \int_{\mathbb{R}^{d}}|G(s-v, x-z)|^{2} d z d v\right. \\
& \left.+\int_{t-\varepsilon}^{s} \int_{\mathbb{R}^{d}} \mathbb{E}\left|\int_{v}^{s} \int_{\mathbb{R}^{d}} G\left(s-r_{1}, y-z_{1}\right) b^{\prime}\left(u\left(r_{1}, z_{1}\right)\right) D_{v, z} u\left(r_{1}, z_{1}\right) d z_{1} d r_{1}\right|^{2} d z d v\right] \\
:= & 2\left(L_{\varepsilon, 1}(s, y)+L_{\varepsilon, 2}(s, y)\right) .
\end{aligned}
$$

With the estimate (16) associated with the Green function $G(t, x)$, we have

$$
\int_{\mathbb{R}^{d}}|G(t-s, y-z)|^{2} d z \leq C^{*}(t-s)^{-\frac{d}{\alpha+\gamma}}
$$

Then

$$
L_{\varepsilon, 1}(s, y) \leq C^{*} \int_{t-\varepsilon}^{s}(s-v)^{-\frac{d}{\alpha+\gamma}} d v \leq C_{5.1 .11} \epsilon^{1-\frac{d}{\alpha+\gamma}}
$$

For the second term $L_{\varepsilon, 2}(s, y)$, we apply the Hölder inequality, the fact that $b^{\prime}$ is bounded and Fubini's theorem, so that we end up with

$$
\begin{aligned}
L_{\varepsilon, 2}(s, y) \leq & C_{5.1 .12} \varepsilon \int_{t-\varepsilon}^{s} \int_{\mathbb{R}^{d}}\left|G\left(s-r_{1}, y-z_{1}\right)\right|^{2} \\
& \times\left(\int_{t-\varepsilon}^{s} \int_{\mathbb{R}^{d}} \mathbb{E}\left(D_{v, z} u\left(r_{1}, z_{1}\right)\right)^{2} d z d v\right) d z_{1} d r_{1} .
\end{aligned}
$$


Then, from (54), (55), (56), (57), we obtain

$$
\begin{aligned}
& \sup _{(\rho, y) \in[t-\varepsilon, s] \times \mathbb{R}^{d}} \mathbb{E}\left(\int_{t-\varepsilon}^{s} \int_{\mathbb{R}^{d}}\left|D_{v, z} u(\rho, y)\right|^{2} d z d v\right) \\
& \leq C_{5.1 .11} \varepsilon^{1-\frac{d}{\alpha+\gamma}}+C_{5.1 .12} \varepsilon \int_{t-\varepsilon}^{s}(s-r)^{-\frac{d}{\alpha+\gamma}} \sup _{(\tau, m) \in[t-\varepsilon, r] \times \mathbb{R}^{d}} \\
& \quad \times \mathbb{E}\left(\int_{t-\varepsilon}^{s} \int_{\mathbb{R}^{d}}\left|D_{v, z} u(\tau, m)\right|^{2} d z d v\right) d r .
\end{aligned}
$$

Now we can conclude by using Gronwall's lemma (for example, Lemma 15 in Dalang [10]).

Furthermore according to Bouleau and Hirsch's criterion, if a random variable $F$ in the space $\mathbb{D}^{1,2}$ satisfies the non-degeneracy condition $\|D F\|_{\mathcal{H}}>0$, a.s., then the law of $F$ is absolutely continuous with respect to the Lebesgue measure.

Proof of Theorem 5.1 We will adopt a technical argument which has been proposed by many authors (see, e.g., Cardon-Weber [9]) to prove Theorem 5.1. It suffices to prove that

$$
\|D u(t, x)\|_{\mathcal{H}}>0
$$

Notice that (see, e.g., Jiang et al. [12])

$$
\|D u\|_{\mathcal{H}}>0 \Leftrightarrow\|D u\|_{L^{2}\left([0, T] \times \mathbb{R}^{d}\right)}>0 .
$$

Hence we only need to prove that $\|D u\|_{L^{2}\left([0, T] \times \mathbb{R}^{d}\right)}>0$ a.s. For $0<\varepsilon<t$, recall (45), we have

$$
\begin{aligned}
\int_{0}^{t} \int_{\mathbb{R}^{d}}\left|D_{r, z} u(t, x)\right|^{2} d z d r & \geq \int_{t-\varepsilon}^{t} \int_{\mathbb{R}^{d}}\left|D_{r, z} u(t, x)\right|^{2} d z d r \\
& \geq C_{5.1 .13}\left(I_{1}(t, x, \varepsilon)-I_{2}(t, x, \varepsilon)\right),
\end{aligned}
$$

where

$$
I_{1}(t, x, \varepsilon)=\int_{t-\varepsilon}^{t} \int_{\mathbb{R}^{d}}|G(t-r, x-z)|^{2} d z d r
$$

and

$$
I_{2}(t, x, \varepsilon)=\int_{t-\varepsilon}^{t} \int_{\mathbb{R}^{d}}\left|\int_{r}^{t} \int_{\mathbb{R}^{d}} G\left(t-r_{1}, x-z_{1}\right) b^{\prime}\left(u\left(r_{1}, z_{1}\right)\right) D_{r, z} u\left(r_{1}, z_{1}\right) d z_{1} d r_{1}\right|^{2} d z d r .
$$

According to (16), there exists a constant $C_{*}>0$ such that

$$
I_{1}(t, x, \varepsilon)=C_{*} \varepsilon^{1-\frac{d}{\alpha+\gamma}} .
$$


By (56) and Lemma 5.1, one gets

$$
\begin{aligned}
\mathbb{E}\left|I_{2}(t, x, \varepsilon)\right| & \leq \int_{t-\varepsilon}^{t} \int_{\mathbb{R}^{d}}\left|G\left(t-r_{1}, x-z_{1}\right)\right| \mathbb{E}\left(\int_{t-\varepsilon}^{r_{1}} \int_{\mathbb{R}^{d}}\left|D_{r, z} u\left(r_{1}, z_{1}\right)\right|^{2} d z d r\right) d z_{1} d r_{1} \\
& \leq C_{5.1 .14} \varepsilon^{1-\frac{d}{\alpha+\gamma}} \int_{t-\varepsilon}^{t} \int_{\mathbb{R}^{d}}\left|G\left(t-r_{1}, x-z_{1}\right)\right| d z_{1} d r_{1} \\
& \leq C_{5.1 .15} \varepsilon^{2-\frac{3 d}{2 \alpha+2 \gamma}} .
\end{aligned}
$$

Then, for each $\varepsilon_{0}>0$, according to (58), (59) and (60),

$$
\begin{aligned}
P\left(\int_{0}^{t} \int_{\mathbb{R}}\left|D_{r, z} u(t, x)\right|^{2} d z d r>0\right) & \geq \sup _{\varepsilon \in\left(0, \varepsilon_{0}\right]} P\left(C_{5.1 .16}\left(I_{1}(t, x, \varepsilon)-I_{2}(t, x, \varepsilon)\right)>0\right) \\
& \geq \sup _{\varepsilon \in\left(0, \varepsilon_{0}\right]} P\left(I_{2}(t, x, \varepsilon) \leq C_{5.1 .17} I_{1}(t, x, \varepsilon)\right) \\
& \geq 1-\inf _{\varepsilon \in\left(0, \varepsilon_{0}\right]}\left\{\frac{1}{C_{5.1 .18} \varepsilon^{1-\frac{d}{\alpha+\gamma}}} \mathbb{E}\left|I_{2}(t, x, \varepsilon)\right|\right\} \\
& \geq 1-\inf _{\varepsilon \in\left(0, \varepsilon_{0}\right]} C_{5.1 .19} \varepsilon^{1-\frac{d}{2 \alpha+2 \gamma}}=1 .
\end{aligned}
$$

Thus the proof of this theorem is complete.

\subsection{Lower and upper bounds for the density}

Let us consider $T>0$ and let $u=\left\{u(t, x),(t, x) \in[0, T] \times \mathbb{R}^{d}\right\}$ be the unique mild solution to Eq. (1). This section is devoted to proving the following result concerning with the Gaussian-type estimates for the density of $u(t, x)$ at any fixed $(t, x) \in[0, T] \times \mathbb{R}^{d}$.

Theorem 5.2 Fix $t \in[0, T]$ and $x \in \mathbb{R}^{d}$. Suppose that Hypothesis 3 is satisfied for some $\eta \in(0,1)$. Moreover, the coefficient $b(\cdot)$ is of class $C^{1}\left(\mathbb{R}^{d}\right)$ and has a bounded Lipschitz continuous derivative. Then the density of the random variable $u(t, x)$ satisfies the following: for almost every $z \in \mathbb{R}$

$$
\frac{\mathbb{E}|u(t, x)-m|}{C_{5.2 .2} t^{2 H}} \exp \left\{-\frac{(z-m)^{2}}{C_{5.2 .1} t^{2 H}}\right\} \leq p(z) \leq \frac{\mathbb{E}|u(t, x)-m|}{C_{5.2 .1} t^{2 H}} \exp \left\{-\frac{(z-m)^{2}}{C_{5.2 .2} t^{2 H}}\right\}
$$

where $m=\mathbb{E} u(t, x)$ and $C_{5.2 .1}$ and $C_{5.2 .2}$ are positive constants depending on $H,\left\|b^{\prime}\right\|_{\infty}, \eta, T$.

Under Hypothesis 3, one can conclude this lemma from the proof of Proposition 3.2.

Lemma 5.2 Let $d<\alpha+\gamma, d \geq 1$ and $T \geq 0$. Then under Hypothesis 3 , we have:

1. There exists a positive constant $k_{1}$ such that for any $t \in[0, T]$

$$
\alpha_{H} \int_{0}^{t} \int_{0}^{t} \int_{\mathbb{R}^{d}} \mathcal{F} G(u, \cdot)(\xi) \overline{\mathcal{F} G(v, \cdot)(\xi)}|u-v|^{2 H-2} \mu(d \xi) d u d v \geq k_{1} t^{2 H}
$$

2. There exists a positive constant $k_{2}$ such that, for any $t \in[0, T]$,

$$
\alpha_{H} \int_{0}^{t} \int_{0}^{t} \int_{\mathbb{R}^{d}} \mathcal{F} G(u, \cdot)(\xi) \overline{\mathcal{F} G(v, \cdot)(\xi)}|u-v|^{2 H-2} \mu(d \xi) d u d v \leq k_{2} t^{2 H}
$$


Remark 5.1 It is worth mentioning that the integrability condition (12) was sufficient for us to prove the existence of density for the solution $u(t, x)$ at any fixed point $(t, x) \in[0, T] \times$ $\mathbb{R}^{d}$. However, as will be made clearer in Lemma 5.2 , we will really need lower and upper bounds of the form (63) and (64) in order to obtain lower and upper bounds for the density of $u(t, x)$ at any fixed $(t, x) \in[0, T] \times \mathbb{R}^{d}$.

Remark 5.2 It is interesting to note that the lower and upper bounds obtained in this proposition did not include the parameter $\alpha$ and $\gamma$.

Theorem 5.2 will be a consequence of Theorem 3.1 in [17] and Proposition 5.1. We use the notation $F=u(t, x)-\mathbb{E} u(t, x)$ and we recall that we will need to find almost sure lower and upper bounds for the random variable $g_{F}(F)$, which is given by

$$
\begin{aligned}
g_{F}(F) & =\int_{0}^{\infty} e^{-\zeta} \mathbb{E}\left[\mathbb{E}^{\prime}\left(\langle D F, \widetilde{D F}\rangle_{\mathcal{H}}\right) \mid F\right] d \zeta \\
& =\int_{0}^{\infty} e^{-\zeta} \mathbb{E}\left[\mathbb{E}^{\prime}\left(\langle D u(t, x), \widetilde{D u(t, x)}\rangle_{\mathcal{H}}\right) \mid F\right] d \zeta
\end{aligned}
$$

where $\widetilde{D F}=(D F)\left(e^{-\zeta} \omega+\sqrt{1-e^{-2 \zeta}} \omega^{\prime}\right)$.

Proposition 5.1 Fix $T>0$ and assume that $d<\alpha+\gamma$ and the function $b(\cdot)$ is of $C^{1}\left(\mathbb{R}^{d}\right)$ with a bounded Lipschitz continuous derivative. Then, for all $t \in[0, T]$, there exist positive constants $C_{5.2 .1}$ and $C_{5.2 .2}$

$$
C_{5.2 .1} t^{2 H} \leq g_{F}(F) \leq C_{5.2 .2} t^{2 H}
$$

In order to prove Proposition 5.1, we will also need the following lemma, whose proof is similar to that of Lemma 5.1, Lemma 4.6 in Nualart and Quer-Sardanyons [19] or Lemma 5 in Nualart and Quer-Sardanyons [20].

Lemma 5.3 For $\delta \in(0,1]$ and assuming $d<\alpha+\gamma$ and Hypothesis 3 holds, there exist two positive constants $C_{5.2 .3}$ and $C_{5.2 .4}$ such that depending on $\left\|b^{\prime}\right\|_{\infty}, \zeta$ and the constant $k_{2}$ in (64) such that

$$
\sup _{(r, y) \in[(1-\delta) t, t] \times \mathbb{R}^{d}} \mathbb{E}\left[\|D u(r, y)\|_{\mathcal{H}\left([(1-\delta) t, t] \times \mathbb{R}^{d}\right)}^{2} \mid F\right] \leq C_{5.2 .3}(\delta t)^{2 H}, \quad \text { a.s. }
$$

and

$$
\sup _{\zeta \geq 1} \sup _{(r, y) \in[(1-\delta) t, t] \times \mathbb{R}^{d}} \mathbb{E}\left[\mathbb{E}^{\prime}\left(\|\widetilde{D u(r, y)}\|_{\mathcal{H}\left([(1-\delta) t, t] \times \mathbb{R}^{d}\right)}^{2}\right) \mid F\right] \leq C_{5.2 .4}(\delta t)^{2 H}, \quad \text { a.s. }
$$

Proof From (45) in Theorem 5.1, and applying the Minkowski inequality, we get

$$
\begin{aligned}
& \|D u(t, x)\|_{\mathcal{H}\left([(1-\delta) t, t] \times \mathbb{R}^{d}\right)} \\
& \leq\|G(t-\cdot, x-*)\|_{\mathcal{H}\left([(1-\delta) t, t] \times \mathbb{R}^{d}\right)} \\
& \quad+\left\|b^{\prime}\right\|_{\infty} \int_{0}^{t} \int_{\mathbb{R}^{d}}|G(t-s, x-y)| \mathbb{E}\left[\|D u(s, y)\|_{\mathcal{H}\left([(1-\delta) t, t] \times \mathbb{R}^{d}\right)}\right] d y d s .
\end{aligned}
$$


As a consequence, we have the following estimate:

$$
\begin{aligned}
\mathbb{E}\left[\|D u(t, x)\|_{\mathcal{H}\left([(1-\delta) t, t] \times \mathbb{R}^{d}\right)} \mid F\right] \\
\leq\|G(t-\cdot, x-*)\|_{\mathcal{H}\left([(1-\delta) t, t] \times \mathbb{R}^{d}\right)} \\
\quad+\left\|f^{\prime}\right\|_{\infty} \int_{0}^{t} \int_{\mathbb{R}^{d}}|G(t-s, x-y)| \mathbb{E}\left[\|D u(s, y)\|_{\mathcal{H}\left([(1-\delta) t, t] \times \mathbb{R}^{d}\right)} \mid F\right] d y d s .
\end{aligned}
$$

Let

$$
Y_{t}:=\sup _{(r, x) \in[0, t] \times \mathbb{R}^{d}} \mathbb{E}\left[\|D u(r, x)\|_{\mathcal{H}\left([(1-\delta) t, t] \times \mathbb{R}^{d}\right)} \mid F\right]
$$

Then according to (64), we have proved that

$$
Y_{t} \leq C_{5.2 .7}(\delta t)^{2 H}+\int_{0}^{t}(t-s)^{-\frac{d}{2(\alpha+\gamma)}} Y_{s} d s
$$

Then a suitable generalization of the Gronwall-type lemma (see, for example, Lemma 15 in Dalang [10]) allows us to conclude the proof. The estimation (68) can be checked using exactly the same arguments.

Proof of Proposition 5.1 We first recall that the Malliavin derivative of $u(t, x),(t, x) \in$ $[0, T] \times \mathbb{R}$ satisfies $D_{v, z} u(s, y) \geq 0$, for all $(v, z) \in[0, T] \times \mathbb{R}^{d}$, a.s. This is because the Malliavin derivative solves the linear equation (45). Let us deal with the proof of (66) in two steps. Our method used here is essentially due to Nualart and Quer-Sardanyons [19] and [20].

Step 1. The lower bound. Fix $\delta \in(0,1]$ and let us first derive the lower bound of $(66)$. Since the Malliavin derivative of $u(t, x)$ is non-negative, Eq. (65) yields

$$
g_{F}(F) \geq \int_{0}^{\infty} e^{-\zeta} \mathbb{E}\left[\mathbb{E}^{\prime}\left(\left\langle D u(t, x),\left.\widetilde{D u(t, x)}\right|_{\mathcal{H}\left([(1-\delta) t, t] \times \mathbb{R}^{d}\right)}\right) \mid F\right] d \xi\right.
$$

By (45), we can decompose the right-hand side of the above (71) in a sum of four terms:

$$
\begin{aligned}
\Theta_{0}(t, x ; \delta)= & \|G(t-\cdot, x-\cdot)\|_{\mathcal{H}\left([(1-\delta) t, t] \times \mathbb{R}^{d}\right)^{\prime}}^{2} \\
\Theta_{1}(t, x ; \delta)= & \mathbb{E}\left[\int_{0}^{t} \int_{\mathbb{R}^{d}} G(t-s, x-y) b^{\prime}(u(s, y))\right. \\
& \left.\times\langle G(t-\cdot, x-\cdot), D u(s, y)\rangle_{\mathcal{H}\left([(1-\delta) t, t] \times \mathbb{R}^{d}\right)} d y d s \mid F\right], \\
\Theta_{2}(t, x ; \delta)= & \int_{0}^{\infty} e^{-\zeta} \mathbb{E}\left[\mathbb { E } ^ { \prime } \left(\int_{0}^{t} \int_{\mathbb{R}^{d}} G(t-s, x-y) b^{\prime}(\widehat{u(s, y))})\right.\right. \\
& \left.\left.\times\langle G(t-\cdot, x-\cdot), \widehat{D u(s, y)}\rangle_{\mathcal{H}\left([(1-\delta) t, t] \times \mathbb{R}^{d}\right)} d y d s \mid F\right)\right] d \sigma, \\
\Theta_{3}(t, x ; \delta)= & \int_{0}^{+\infty} e^{-\zeta} \mathbb{E}\left[\mathbb{E}^{\prime} \int_{0}^{t} \int_{\mathbb{R}^{d}} \int_{0}^{t} \int_{\mathbb{R}^{d}} G(t-s, x-y) b^{\prime}(u(s, y)) G(t-r, x-z)\right. \\
& \left.\left.\times b^{\prime}(\widehat{u(r, z)})\langle D u(s, y), \widehat{D u(r, z)}\rangle_{\mathcal{H}\left([(1-\delta) t, t] \times \mathbb{R}^{d}\right)} d r d s d y d z \mid F\right)\right] d \sigma .
\end{aligned}
$$


Firstly we notice that with $d<\alpha+\gamma$ and (63) in Lemma 5.2,

$$
\Theta_{0}(t, x ; \delta) \geq k_{1}(\delta t)^{2 H}
$$

Thus we can write

$$
g_{F}(F) \geq k_{1}(\delta t)^{2 H}-\left|\Theta_{1}(t, x ; \delta)+\Theta_{2}(t, x ; \delta)+\Theta_{3}(t, x ; \delta)\right| .
$$

Thus we will need to obtain the upper bounds for the terms $\Theta_{i}(t, x ; \delta), i=1,2,3$. We apply Fubini's theorem, the boundedness of $b^{\prime}$, the estimate (64) in Lemma 5.2 and the bound (67) in Lemma 5.3. Then we have the following estimate:

$$
\begin{aligned}
& \left|\Theta_{1}(t, x ; \delta)\right| \\
& \leq C_{5.2 .9}\|G(t-\cdot, x-\cdot)\|_{\mathcal{H}\left([(1-\delta) t, t] \times \mathbb{R}^{d}\right)}\left\|b^{\prime}\right\|_{\infty} \\
& \quad \times\left[\int_{(1-\delta) t}^{t} \int_{\mathbb{R}^{d}}|G(t-s, x-y)| \mathbb{E}\|D u(s, y)\|_{\mathcal{H}\left([(1-\delta) t, t] \times \mathbb{R}^{d}\right)} d y d s \mid F\right] \\
& \leq C_{5.2 .10}\|G(t-\cdot, x-\cdot)\|_{\mathcal{H}\left([(1-\delta) t, t] \times \mathbb{R}^{d}\right)}\left\|b^{\prime}\right\|_{\infty} \\
& \quad \times \sup _{(s, y) \in[(1-\delta) t, t] \times \mathbb{R}^{d}} \mathbb{E}\|D u(s, y)\|_{\mathcal{H}\left([(1-\delta) t, t] \times \mathbb{R}^{d}\right)} \int_{(1-\delta) t}^{t} \int_{\mathbb{R}^{d}}|G(t-s, x-y)| d y d s \\
& \leq C_{5.2 .11}(\delta t)^{2 H+1-\frac{d}{2(\alpha+\gamma)}} .
\end{aligned}
$$

In order to get an upper bound for $\left|\Theta_{2}(t, x ; \delta)\right|$, one can proceed using exactly the same arguments as for $\left|\Theta_{1}(t, x ; \delta)\right|$, but apply (68) in Lemma 5.3 instead of (67) in Lemma 5.3. Hence one obtains

$$
\left|\Theta_{2}(t, x ; \delta)\right| \leq C_{5.2 .14}(\delta t)^{2 H+1-\frac{d}{2(\alpha+\gamma)}} .
$$

Let us finally estimate $\left|\Theta_{3}(t, x ; \delta)\right|$. For this, we apply Fubini's theorem, the fact that $b^{\prime}$ is bounded, the Cauchy-Schwartz inequality, and we finally invoke Lemma 5.3,

$$
\begin{aligned}
& \left|\Theta_{3}(t, x ; \delta)\right| \\
& \quad \leq C_{5.2 .15}\left\|f^{\prime}\right\|_{\infty} \int_{0}^{+\infty} e^{-\zeta}\left[\int_{(1-\delta) t}^{t} \int_{\mathbb{R}^{d}} \int_{(1-\delta) t}^{t} \int_{\mathbb{R}^{d}} G(t-s, x-y) G(t-\bar{s}, x-\bar{y})\right. \\
& \left.\quad \times\left(\mathbb{E}\left[\|D u(s, y)\|_{\mathcal{H}\left([(1-\delta) t, t] \times \mathbb{R}^{d}\right)} \mathbb{E}^{\prime}\left(\|D \widehat{D(\bar{s}, \bar{y})}\|_{\mathcal{H}\left([(1-\delta) t, t] \times \mathbb{R}^{d}\right)}\right) \mid F\right]\right) d y d s d \bar{y} d \bar{s}\right] d \sigma .
\end{aligned}
$$

At this point, we apply the Cauchy-Schwartz inequality with respect to the conditional expectation with respect to $F$. One can use the bounds (67) and (68) in Lemma 5.3 and obtain

$$
\left|\Theta_{3}(t, x ; \delta)\right| \leq C_{5.2 .17}(\delta t)^{2 H+1-\frac{d}{\alpha+\gamma}} .
$$

Eventually, plugging the bounds (77), (78), (79) into (76), we have with $d<\alpha+\gamma$

$$
g_{F}(F) \geq k_{1}(\delta t)^{2 H}-\left[C_{5.2 .19}(\delta t)^{2 H+1-\frac{d}{2(\alpha+\gamma)}}+C_{5.2 .17}(\delta t)^{2 H+1-\frac{d}{\alpha+\gamma}}\right] .
$$


Hence we have

$$
g_{F}(F) \geq t^{2 H}\left[k_{1} \delta^{2 H}-\delta^{2 H}\left(C_{5.2 .19}(\delta T)^{1-\frac{d}{2(\alpha+\gamma)}}+C_{5.2 .17}(\delta T)^{1-\frac{d}{\alpha+\gamma}}\right)\right]
$$

Hence if we assume that $\delta<1 \wedge \frac{1}{T}$, it only remains to choose a positive quantity $\delta$ sufficiently small such that the quantity $\delta^{2 H}\left[k_{1}-\left(C_{5.2 .19}(\delta T)^{1-\frac{d}{2(\alpha+\gamma)}}+C_{5.2 .17}(\delta T)^{1-\frac{d}{\alpha+\gamma}}\right)\right]$ is strictly positive, then we can write

$$
g_{F}(F) \geq C_{5.2 .1} t^{2 H}
$$

Thus, the lower bound in (66) has been proved.

Step 2. The upper bound. The upper bound in (66) is almost an immediate consequence of the computations which we have just performed for the lower bound. More precisely, according to $g_{F}(F)$ and the considerations in the first part of the proof, we have the following:

$$
g_{F}(F) \leq \sum_{i=0}^{3} \Theta_{i}(t, s ; \delta),
$$

where we notice that we have substituted $\delta$ by 1 in $\Theta_{i}(t, x ; \delta), i=0,1,2,3$. We have already seen that, for $i=1,2$,

$$
\left|\Theta_{i}(t, x ; 1)\right| \leq C_{5.2 .14} t^{2 H+1-\frac{d}{2(\alpha+\gamma)}}
$$

and

$$
\left|\Theta_{3}(t, x ; 1)\right| \leq C_{5.2 .17} t^{2 H+1-\frac{d}{\alpha+\gamma}}
$$

So we just need to bound $\Theta_{0}(t, x ; 1)$, which follows directly from (64). Thus

$$
g_{F}(F) \leq k_{3} t^{2 H}+2 C_{5.2 .14} t^{2 H+1-\frac{d}{2(\alpha+\gamma)}}+C_{5.2 .17} t^{2 H+1-\frac{d}{\alpha+\gamma}} .
$$

Therefore we conclude that

$$
g_{F}(F) \leq C_{5.2 .2} t^{2 H}
$$

with the positive constants $C_{5.2 .2}$ depending on $T$. Therefore the proof of this proposition is complete.

Proof of Theorem 5.2 For any fixed $(t, x) \in[0, T] \times \mathbb{R}$, we know that the random variable $F=u(t, x)-\mathbb{E}(u(t, x))$ is centered and belongs to $\mathbb{D}^{1,2}$ and by (66); we have $0<C_{5.2 .1} t^{2 H} \leq$ $g_{F}(F)$ for all $t \in[0, T]$. We then apply Theorem 3.1 and Corollary 3.3 in Nourdin and Viens [17], and find that the probability density $\rho: \mathbb{R} \mapsto \mathbb{R}$ of the random variable $F$ is given by

$$
\rho(z)=\frac{\mathbb{E}|u(t, x)-\mathbb{E}(u(t, x))|}{2 g_{F}(z)} \exp \left\{-\int_{0}^{z} \frac{y}{g_{F}(y)} d y\right\}
$$


for almost every $z \in \mathbb{R}$. Then the density $p$ of the random variable $u(t, x)$ satisfies

$$
p(z)=\frac{\mathbb{E}|u(t, x)-\mathbb{E}(u(t, x))|}{2 g_{F}(z-\mathbb{E}(u(t, x)))} \exp \left\{-\int_{0}^{z-\mathbb{E}(u(t, x))} \frac{y}{g_{F}(y)} d y\right\} .
$$

In order to conclude the proof, we only need to apply the bounds obtained in Proposition 5.1 to $(80)$.

\section{Acknowledgements}

The authors thank their friends and reviewers for their valuable suggestions, which substantially improved the quality of the paper.

\section{Funding}

Not applicable.

\section{Availability of data and materials}

Not applicable.

\section{Competing interests}

The authors declare that they have no competing interests.

\section{Authors' contributions}

The authors declare that this study was accomplished in collaboration with the same responsibility. All authors read and approved the final manuscript.

\section{Publisher's Note}

Springer Nature remains neutral with regard to jurisdictional claims in published maps and institutional affiliations.

Received: 8 July 2020 Accepted: 10 February 2021 Published online: 04 March 2021

\section{References}

1. Angulo, J.M., Anh, V.V., McVinish, R., Ruiz-Medina, M.D.: Fractional kinetic equations driven by Gaussian or infinitely divisible noise. Adv. Appl. Probab. 37, 366-392 (2005)

2. Angulo, J.M., Ruiz-Medina, M.D., Anh, V.V., Grecksch, W.: Fractional diffusion and fractional heat equation. Adv. Appl. Probab. 32, 1077-1099 (2000)

3. Anh, V.V., Angulo, J.M., Ruiz-Medina, M.D.: Possible long-range dependence in fractional random fields. J. Stat. Plan. Inference 80, 95-110 (1999)

4. Anh, V.V., Leonenko, N.N.: Spectral analysis of fractional kinetic equations with random data. J. Stat. Phys. 104 1349-1387 (2001)

5. Anh, V.V., Leonenko, N.N., McVinish, R.: Models for fractional Riesz-Bessel motion and related processes. Fractals 9 329-346 (2001)

6. Anh, V.V., Leonenko, N.N., Ruiz-Medina, M.D.: Space-time fractional stochastic equations on regular bounded open domains. Fract. Calc. Appl. Anal. 19, 1161-1199 (2016)

7. Balan, R.M., Tudor, C.A.: The stochastic heat equation with fractional-colored noise: existence of the solution. ALEA Lat. Am. J. Probab. Math. Stat. 4, 57-87 (2008); Latin Amer. J. Probab. Math. Stat. 6, 343-347 (2009) (erratum)

8. Balan, R.M., Tudor, C.A.: The stochastic wave equation with fractional noise: a random field approach. Stoch. Process. Appl. 120, 2468-2494 (2010)

9. Cardon-Weber, C.: Cahn-Hilliard stochastic equation: existence of the solution and its density. Bernoulli 7, 777-816 (2000)

10. Dalang, R.: Extending the martingale measure stochastic integral with applications to spatially homogeneous SPDE's. Electron. J. Probab. 4(6), 1-29 (1999)

11. Hu, Y., Huang, J., Nualart, D., Tindel, S.: Stochastic heat equations with general multiplicative Gaussian noises: Hölder continuity and intermittency. Electron. J. Probab. 20(55), 1-50 (2015)

12. Jiang, Y., Wei, T., Zhou, X.: Stochastic generalized Burgers equations driven by fractional noises. J. Differ. Equ. 252 , 1934-1961 (2012)

13. Jiménez, J.: Hyperviscous vortices. J. Fluid Mech. 279, 169-176 (1994)

14. Liu, J., Yan, L.: Solving a nonlinear fractional stochastic partial differential equation with fractional noise. J. Theor. Probab. 29, 307-347 (2016)

15. Márquez-Carreras, D.: Generalized fractional kinetic equations: another point of view. Adv. Appl. Probab. 41, 893-910 (2009)

16. Márquez-Carreras, D.: Generalized stochastic heat equation. In: Malliavin Calculus and Stochastic Analysis: A Festschrift in Honor of David Nualart. Springer Proceedings in Mathematics and Statistics, vol. 34, pp. $281-297$. Springer, New York (2013) https://doi.org/10.1007/978-1-4614-5906-4

17. Nourdin, I., Viens, F.G.: Density formula and concentration inequalities with Malliavin calculus. Electron. J. Probab. 14, 2287-2309 (2009)

18. Nualart, D.: The Malliavin Calculus and Related Topics, 2nd edn. Springer, Berlin (2006) 
19. Nualart, D., Quer-Sardanyons, L.: Gaussian density estimates for solutions to quasi-linear stochastic partial differential equations. Stoch. Process. Appl. 119, 3914-3938 (2009)

20. Nualart, D., Quer-Sardanyons, L.: Optimal Gaussian density estimates for a class of stochastic equations with additive noise. Infin. Dimens. Anal. Quantum Probab. Relat. Top. 14, 25-34 (2011)

21. Walsh, J.B.: A stochastic model of neural response. Adv. Appl. Probab. 13, 231-281 (1981)

22. Walsh, J.B.: An introduction to stochastic partial differential equations. In: Ecole d'été de Probabilités de St. Flour XIV. Lect. Notes in Math., vol. 1180, pp. 266-439. Springer, Berlin (1986)

23. Zhang, T., Zheng, W.: SPDEs driven by space-time white noises in high dimensions: absolute continuity of the law and convergence of solutions. Stoch. Stoch. Rep. 75, 103-128 (2003)

Submit your manuscript to a SpringerOpen ${ }^{\odot}$ journal and benefit from:

- Convenient online submission

- Rigorous peer review

- Open access: articles freely available online

- High visibility within the field

- Retaining the copyright to your article

Submit your next manuscript at $\gg$ springeropen.com 